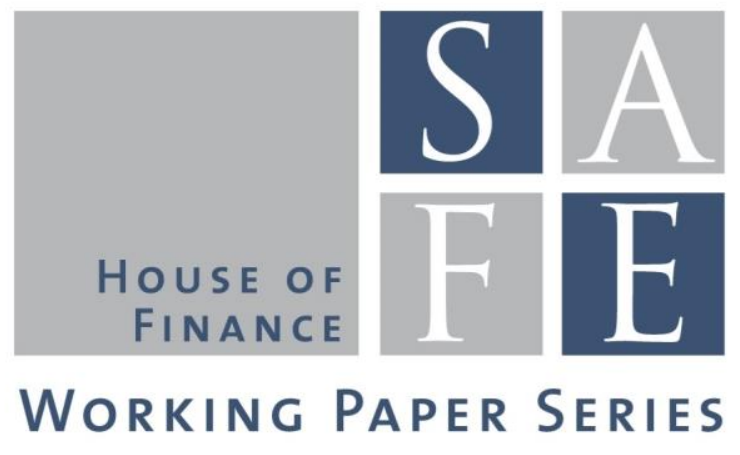

Holger Kraft - Claus Munk - Farina Weiss

\title{
Predictors and Portfolios over the Life Cycle
}

SAFE Working Paper No. 139

\section{SAFE I Sustainable Architecture for Finance in Europe}

A cooperation of the Center for Financial Studies and Goethe University Frankfurt 


\section{Non-Technical Summary}

A sizeable literature has investigated the predictability of stock returns, and several papers have quantified the impact of return predictability on stock-bond asset allocation decisions in a standard utility maximization framework. For households, portfolio decisions should be seen in a life-cycle perspective incorporating human capital and real estate, the dominant assets for many households (Campbell 2006). The ability to predict house prices and labor income is potentially as important for households as stock market predictability.

Our study is the first to study predictability in the joint dynamics of stock prices, house prices, and income, which is essential for household decisions. Due to the contemporaneous correlation between the prices and income on one hand and the predictors on the other hand, the model produces a rich longer-run correlation structure between stock prices, house prices, and labor income that, for example, allows expected stock returns to be correlated with house prices or labor income.

In this paper we estimate empirically the joint dynamics of stock prices, house prices, and labor income. We show that the net corporate payout yield predicts both the stock market index and house prices, whereas the log home rent-price ratio predicts both house prices and labor income.

We embed the estimated dynamics in a rich model of household decisions involving consumption of perishable goods and housing services, unspanned labor income, stochastic house prices, home renting and owning, stock investments, and portfolio constraints. We assume in our baseline case a modestly risk-averse agent having a 35-year working period and a 15-year retirement period. Within this model we show that, by optimally conditioning decisions on the predictors, households can (a) generate larger average returns on investments and thus larger consumption and (b) reduce the likelihood of ending up with very low consumption and thus very low utility.

As strategies are chosen to maximize expected utility, along a given sample path the strategy derived assuming predictability is not guaranteed to perform better than the strategy ignoring predictability. However, we show that along the observed path in our 1960-2010 period, all cohorts of agents entering our model in the years 1961 to 1976 would have benefited from conditioning decisions on the predictors, especially the 1970-1976 cohorts since one or both predictors have been sizeable in most years since 1970 . 


\title{
Predictors and Portfolios over the Life Cycle
}

\author{
Holger Kraft $^{\mathrm{a}} \quad$ Claus Munk ${ }^{\mathrm{b}} \quad$ Farina Weiss $^{\mathrm{c}}$
}

Current version: May 31, 2016

\begin{abstract}
We show that the net corporate payout yield predicts both the stock market index and house prices and that the log home rent-price ratio predicts both house prices and labor income growth. We incorporate the predictability in a rich life-cycle model of household decisions involving consumption of both perishable goods and housing services, stochastic and unspanned labor income, stochastic house prices, home renting and owning, stock investments, and portfolio constraints. We find that households can significantly improve their welfare by optimally conditioning decisions on the predictors. For a modestly risk-averse agent with a 35-year working period and a 15year retirement period, the present value of the higher average life-time consumption amounts to roughly $\$ 179,000$ (assuming both an initial wealth and an initial annual income of $\$ 20,000$ ), and the certainty equivalent gain is around $5.5 \%$ of total wealth (financial wealth plus human capital). Furthermore, every cohort of agents in our model would have benefited from applying predictor-conditional strategies along the realized time series over our 1960-2010 data period.
\end{abstract}

Keywords: Return predictability, human capital, housing, investments, welfare

JEL subject codes: G11, D91, D14

\footnotetext{
${ }^{a}$ Faculty of Economics and Business Administration, Goethe University, Frankfurt am Main, Germany. E-mail: holgerkraft@finance.uni-frankfurt.de

b Department of Finance, Copenhagen Business School, Denmark. E-mail: cm.fi@cbs.dk

${ }^{c}$ Faculty of Economics and Business Administration, Goethe University, Frankfurt am Main, Germany. E-mail: farina.weiss@hof.uni-frankfurt.de

We are grateful for comments and suggestions from participants at the Netspar International Pension Workshop (Frankfurt) as well as seminars at the University of Zürich and Aalto University School of Business (Helsinki), and in particular from the Netspar discussant Frank de Jong. Holger Kraft and Farina Weiss gratefully acknowledges financial support by Deutsche Forschungsgemeinschaft (DFG) as well as the Center of Excellence SAFE, funded by the State of Hessen initiative for research LOEWE.
} 


\title{
Predictors and Portfolios over the Life Cycle
}

\begin{abstract}
We show that the net corporate payout yield predicts both the stock market index and house prices and that the log home rent-price ratio predicts both house prices and labor income growth. We incorporate the predictability in a rich life-cycle model of household decisions involving consumption of both perishable goods and housing services, stochastic and unspanned labor income, stochastic house prices, home renting and owning, stock investments, and portfolio constraints. We find that households can significantly improve their welfare by optimally conditioning decisions on the predictors. For a modestly risk-averse agent with a 35-year working period and a 15year retirement period, the present value of the higher average life-time consumption amounts to roughly $\$ 179,000$ (assuming both an initial wealth and an initial annual income of $\$ 20,000$ ), and the certainty equivalent gain is around $5.5 \%$ of total wealth (financial wealth plus human capital). Furthermore, every cohort of agents in our model would have benefited from applying predictor-conditional strategies along the realized time series over our 1960-2010 data period.
\end{abstract}

Keywords: Return predictability, human capital, housing, investments, welfare

JEL subject codes: G11, D91, D14 


\section{Contents}

1 Introduction $\quad 1$

2 The joint dynamics of stock prices, house prices, and income 3

2.1 Our main model . . . . . . . . . . . . . . . . . . . . . . 4

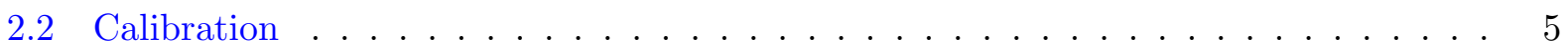

3 The decision problem of a consumer-investor $\quad 9$

3.1 Formulation of the problem $\ldots \ldots \ldots \ldots \ldots$

3.2 Solution method . . . . . . . . . . . . . . . . . . . . 12

4 Illustration and discussions of results $\quad 15$

4.1 Optimal decisions . . . . . . . . . . . . . . . . . . . 16

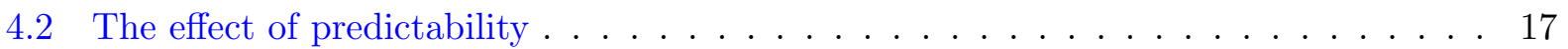

4.3 Historical simulations . . . . . . . . . . . . . . . . . . . . 21

5 Conclusion $\quad 23$

$\begin{array}{ll}\text { A Details of the calibration } & 26\end{array}$

B Details on the numerical method $\quad 26$

B.1 Artificial markets . . . . . . . . . . . . . . . . . 26

B.2 Human capital in computable artificial markets . . . . . . . . . . . . . . . . . . 29

B.3 Optimality in computable artificial markets . . . . . . . . . . . . . . . . 32

B.4 Upper bound on obtainable utility . . . . . . . . . . . . . . . . . . . 41

B.5 Promising feasible strategies for the true problem . . . . . . . . . . . . 41

B.6 Solution in special cases . . . . . . . . . . . . . . . . . . . . 43

$\begin{array}{ll}\text { References } & 44\end{array}$ 


\section{Introduction}

A sizeable literature has investigated the predictability of stock returns, and several papers have quantified the impact of return predictability on stock-bond asset allocation decisions in a standard utility maximization framework. For households, portfolio decisions should be seen in a life-cycle perspective incorporating human capital and real estate, the dominant assets for many households (Campbell 2006). The ability to predict house prices and labor income is potentially as important for households as stock market predictability. In this paper we estimate empirically the joint dynamics of stock prices, house prices, and labor income. We show that the net corporate payout yield predicts both the stock market index and house prices, whereas the log home rent-price ratio predicts both house prices and labor income. ${ }^{1}$

We embed the estimated dynamics in a rich model of household decisions involving consumption of perishable goods and housing services, unspanned labor income, stochastic house prices, home renting and owning, stock investments, and portfolio constraints. Within this model we show that, by optimally conditioning decisions on the predictors, households can (a) generate larger average returns on investments and thus larger consumption and (b) reduce the likelihood of ending up with very low consumption and thus very low utility. In our baseline case for a modestly riskaverse agent having a 35-year working period and a 15-year retirement period, the present value of the higher average life-time consumption amounts to roughly $\$ 179,000$ (assuming both an initial financial wealth and an initial annual income of $\$ 20,000$ ), and the certainty equivalent gain of applying predictor-dependent strategies is around $5.5 \%$ of total wealth (financial wealth plus human capital). As strategies are chosen to maximize expected utility, along a given sample path the strategy derived assuming predictability is not guaranteed to perform better than the strategy ignoring predictability. However, we show that all cohorts of agents entering our model in the years 1961 to 1976 would have benefited from conditioning decisions on the predictors, especially the 1970-1976 cohorts since one or both predictors have been sizeable in most years since 1970 .

We estimate the joint dynamics of stock prices, house prices, and labor income based on aggregate, annual 1960-2010 U.S. data for the CRSP value-weighted stock market portfolio, the national Case-Shiller home price index, and the disposable income per capita (all series are inflation adjusted). We capture predictability by allowing expected growth rates to depend linearly on potential predictors. After considering various predictor candidates, we find that the net corporate

\footnotetext{
${ }^{1}$ The net payout of a company in a given period equals the dividends plus equity repurchases less equity issuances.
} 
payout yield positively predicts stock returns and negatively predicts house price growth, and that the log of the home rent-price ratio negatively predicts house price growth and positively predicts labor income growth. The predictive power of the net payout yield on stock returns is known from Boudoukh, Michaely, Richardson, and Roberts (2007), but its relation to house price growth rates has not been established before. Likewise, the rent-price ratio is known to predict house prices (Himmelberg, Mayer, and Sinai 2005; Plazzi, Torous, and Valkanov 2010), but we document its ability to predict income growth. More importantly, our study is the first to study predictability in the joint dynamics of stock prices, house prices, and income, which is essential for household decisions. Due to the contemporaneous correlation between the prices and income on one hand and the predictors on the other hand, the model produces a rich longer-run correlation structure between stock prices, house prices, and labor income that, for example, allows expected stock returns to be correlated with house prices or labor income. $^{2}$

Our paper builds on the large literature on stock return predictability which reports that expected stock returns vary with such variables as the price-earnings ratio (Campbell and Shiller 1988), the net payout yield (Boudoukh, Michaely, Richardson, and Roberts 2007), past stock returns (Fama and French 1988; Moskowitz, Ooi, and Pedersen 2012), or short-term interest rates (Ang and Bekaert 2007). Koijen and van Nieuwerburgh (2011) survey this literature. The implications for stock-bond asset allocation has been explored by Kim and Omberg (1996), Campbell and Viceira (1999), Barberis (2000), and Wachter (2002) in stylized models disregarding housing and income. Since expected stock returns vary counter cyclically in these models, intertemporal hedging considerations lead to an increased demand for stocks, although the quantitative effects are typically found to be modest. However, it is well known by now that optimal portfolios change substantially with the inclusion of labor income or housing, so the impact of stock return predictability on household portfolios should be explored in richer models. Furthermore, the predictability of house prices or labor income may also affect optimal portfolios.

Cocco, Gomes, and Maenhout (2005) showed that a labor income process calibrated to life-cycle data is more bond-like than stock-like and thus induces agents to invest a large share of financial wealth in stocks, in particular early in life where human capital dominates. Two papers extend their study by allowing the expected income growth to depend on a business cycle variable, either the level of the short-term interest rate level (Munk and Sørensen 2010) or the stock market dividend

\footnotetext{
${ }^{2}$ The housing collateral ratio and the ratio of aggregate labor income to aggregate consumption are also reported to help predicting stock returns (Lustig and van Nieuwerburgh 2005; Santos and Veronesi 2006), which supports our assumption that the expected stock return can be correlated with house prices and labor income.
} 
yield (Lynch and Tan 2011), but these papers ignore housing aspects.

Only few papers derive life-cycle consumption and investment strategies in settings capturing both human capital, housing, and investment risk. Assuming for tractability a perfect correlation between house prices and aggregate income shocks, Cocco (2005) concludes that house price risk crowds out stock holdings and can therefore help in explaining limited stock market participation. Yao and Zhang (2005) generalize Cocco's setting to an imperfect house-income correlation and endogenize the renting/owning decision. They find that home-owners invest less in stocks than home-renters, which confirms that housing risk crowds out stock market risk. ${ }^{3}$ In our more general setting, we also find that the optimal stock investment is zero or low for many young households.

While several papers find evidence of predictability in real estate prices (Case and Shiller 1990, Poterba 1991, Malpezzi 1999, Ghysels, Plazzi, Valkanov, and Torous 2013), only few papers discuss the implications for household decisions. Fischer and Stamos (2013) solve a life-cycle utility maximization problem assuming that expected housing returns depend on realized past returns. We add time-varying drift rates in stocks and income, and we allow the drift rates to be correlated with the levels of stock, house price, and income, which can substantially affect the magnitude and risk characteristics of human capital and therefore optimal investment and consumption decisions. Corradin, Fillat, and Vergara-Alert (2014) show that predictability in house prices causes house [stock] investments to be increasing [decreasing] in the current expected house price growth. Even with a relatively high risk aversion, they report significant stock investments.

The remainder of the paper is organized as follows. Section 2 sets up and estimates the dynamic model of stock prices, house prices, and labor income. In Section 3 we formulate the life-cycle utility maximization problem of an individual consumer-investor and explains how we solve it. Section 4 presents and discusses various aspects of the derived life-cycle consumption and investment behavior. Finally, Section 5 summarizes the paper.

\section{The joint dynamics of stock prices, house prices, and income}

This section presents our model for the joint dynamics of labor income, stock prices, and house prices, and calibrates it to U.S. data. We use a continuous-time formulation since this facilitates the derivation of the optimal consumption and investment decisions in subsequent sections.

\footnotetext{
${ }^{3}$ van Hemert (2010) generalizes further by allowing for stochastic variations in interest rates and thereby introducing a role for bonds, but his focus is on the interest rate exposure and mortgage choice over the life cycle.
} 


\subsection{Our main model}

The time $t$ level of the stock market index is denoted by $S_{t}$, the unit house price by $H_{t}$, and the labor income rate by $L_{t}$. Our main models assumes that the dynamics of these variables are

$$
\begin{aligned}
\frac{d S_{t}}{S_{t}} & =\left(r+\mu_{S}+\chi_{S} x_{t}\right) d t+\sigma_{S} d B_{S t}, \\
\frac{d H_{t}}{H_{t}} & =\left(r+\mu_{H}+\chi_{H x} x_{t}+\chi_{H y} y_{t}\right) d t+\sigma_{H}\left(\rho_{H S} d B_{S t}+\hat{\rho}_{H} d B_{H t}\right), \\
\frac{d L_{t}}{L_{t}} & =\left(\mu_{L}(t)+\bar{\chi}_{L}(t) y_{t}\right) d t+\bar{\sigma}_{L}(t)\left(\rho_{L S} d B_{S t}+\hat{\rho}_{L H} d B_{H t}+\hat{\rho}_{L} d B_{L t}\right) .
\end{aligned}
$$

Here $x$ and $y$ are the predictors with $x_{t}$ represented by the corporate net payout yield and $y_{t}$ by the log of the home rent-price ratio (some alternatives are discussed below). Both predictors have been detrended and are assumed to vary around zero according to the processes

$$
\begin{aligned}
& d x_{t}=-\kappa_{x} x_{t} d t+\sigma_{x}\left(\rho_{x S} d B_{S t}+\hat{\rho}_{x H} d B_{H t}+\hat{\rho}_{x L} d B_{L t}+\hat{\rho}_{x} d B_{x t}\right), \\
& d y_{t}=-\kappa_{y} y_{t} d t+\sigma_{y}\left(\rho_{y S} d B_{S t}+\hat{\rho}_{y H} d B_{H t}+\hat{\rho}_{y L} d B_{L t}+\hat{\rho}_{y x} d B_{x t}+\hat{\rho}_{y} d B_{y t}\right),
\end{aligned}
$$

where $B_{S}, B_{H}, B_{L}, B_{x}, B_{y}$ are independent standard Brownian motions. All instantaneous correlations are constant. We let $\rho_{H S}=\rho_{S H}$ denote the instantaneous correlation between stock price and the house price and use similar notation for the other pairs of processes. In addition, define

$$
\begin{array}{cll}
\hat{\rho}_{H}=\sqrt{1-\rho_{H S}^{2}}, & \hat{\rho}_{L H}=\frac{\rho_{L H}-\rho_{L S} \rho_{H S}}{\hat{\rho}_{H}}, & \hat{\rho}_{L}=\sqrt{1-\rho_{L S}^{2}-\hat{\rho}_{L H}^{2}}, \\
\hat{\rho}_{x H}=\frac{\rho_{x H}-\rho_{x s} \rho_{H S}}{\hat{\rho}_{H}}, & \hat{\rho}_{x L}=\frac{\rho_{x L}-\rho_{x S} \rho_{L S}-\hat{\rho}_{x H} \hat{\rho}_{L H}}{\hat{\rho}_{L}}, & \hat{\rho}_{x}=\sqrt{1-\rho_{x S}^{2}-\hat{\rho}_{x H}^{2}-\hat{\rho}_{x L}^{2}}, \\
\hat{\rho}_{y H}=\frac{\rho_{y H}-\rho_{y s} \rho_{H S}}{\hat{\rho}_{H}}, & \hat{\rho}_{y L}=\frac{\rho_{y L}-\rho_{y S} \rho_{L S}-\hat{\rho}_{y H} \hat{\rho}_{L H}}{\hat{\rho}_{L}}, & \hat{\rho}_{y}=\sqrt{1-\rho_{y S}^{2}-\hat{\rho}_{y H}^{2}-\hat{\rho}_{y L}^{2}-\hat{\rho}_{y x}^{2}},
\end{array}
$$

and

$$
\hat{\rho}_{y x}=\frac{\rho_{y x}-\rho_{y S} \rho_{x S}-\hat{\rho}_{y H} \hat{\rho}_{x H}-\hat{\rho}_{y L} \hat{\rho}_{x L}}{\hat{\rho}_{x}} .
$$

By construction $\mu_{S}$ and $\mu_{H}$ are the long-term average expected growth rates of the stock and house prices per year in excess of the risk-free interest rate $r$, which is assumed constant. The stock price and house price volatilities $\sigma_{S}$ and $\sigma_{H}$ are also constant. The stock pays a constant dividend yield of $\bar{D}$ so that the total dividends paid out over a short interval $[t, t+d t]$ is $\bar{D} S_{t} d t$. The parameters $\kappa_{x}$ and $\sigma_{x}$ denote the speed of mean reversion and the volatility of the net payout 
yield. Similarly for $\kappa_{y}$ and $\sigma_{y}$. Increments to $x$ and $y$ are correlated with increments to $S, H$, and $L$, which implies that the longer-term relations between $S, H$, and $L$ can be markedly different from the short-term relations, cf. the discussion below.

\subsection{Calibration}

We estimate the above dynamics on time series of the stock market index, the national Case-Shiller home price index, and aggregate labor income. In this estimation we assume $\mu_{L}, \bar{\chi}_{L}$, and $\sigma_{L}$ are constant. When embedding the dynamics in the life-cycle decision problem in subsequent sections, we adjust the estimates of the house price and income volatilities to be more representative of individual house prices and labor income, and we allow for age dependence in $\mu_{L}, \bar{\chi}_{L}$, and $\sigma_{L}$.

We use annual U.S. data for stock prices, house prices and aggregate labor income starting in 1960 (where available data on the home rent-price ratio begins) and ending in 2010 (where available data on net payout yield ends). As stock market data, we use returns on the CRSP value-weighted market portfolio inclusive of the NYSE, AMEX, and NASDAQ markets (cum dividend). The riskfree asset is estimated from the Treasury bill yield provided by the Risk Free File on CRSP Bond tape. The house price is represented by the national Case-Shiller home price index with data taken from Robert Shiller's homepage. ${ }^{4}$ From the National Income and Product Accounts (NIPA) tables published by the Bureau of Economic Analysis of the U.S. Department of Commerce, we obtain quarterly U.S. data for aggregated disposable personal income (per capita). The annual returns are computed from quarterly data. To obtain real values, all time-series are deflate using the consumer price index (CPI) taken from CRSP. Figure 1 depicts the time series of the stock price index, per capita labor income, and the national home price index.

As suggested by Boudoukh et al. (2007) we use the log of the sum of 0.1 and the net payout yield as our $x$-variable (for simplicity, we refer to $x$ as the net payout yield in the following), and Professor Michael Roberts supplies the data until 2010 on his homepage. ${ }^{5}$ We use the net payout yield for nonfinancials, but obtain very similar results when including all firms. The data for the rent-price ratio is described in Davis, Lehnert, and Martin (2008) and is downloaded from the homepage of the Lincoln Institute of Land Policy. ${ }^{6}$ Since the $\log$ rent-price ratio is nonstationary but integrated of order one, we use the difference in the log rent-price ratio. To avoid that extreme outliers significantly affect the estimation, we winsorize $x$ at the $4 \%$ level and $y$ at the $2 \%$ level.

\footnotetext{
${ }^{4}$ http://www .econ.yale.edu/\$\sim\$shiller/data.htm

${ }^{5}$ http://finance.wharton. upenn. edu/ mrrobert/

${ }^{6}$ http://www. lincolninst.edu/resources/
} 
Figure 1: Historical evolution of stock market, house prices, and income per capita in the U.S. The stock market is represented by the CRSP value-weighted market portfolio inclusive of the NYSE, AMEX, and NASDAQ markets (cum dividend). The house prices uses the national CaseShiller home price index. Income is disposable labor income per capita from NIPA. All time series are standardized to 100 in 1960 and inflation-adjusted using CPI.

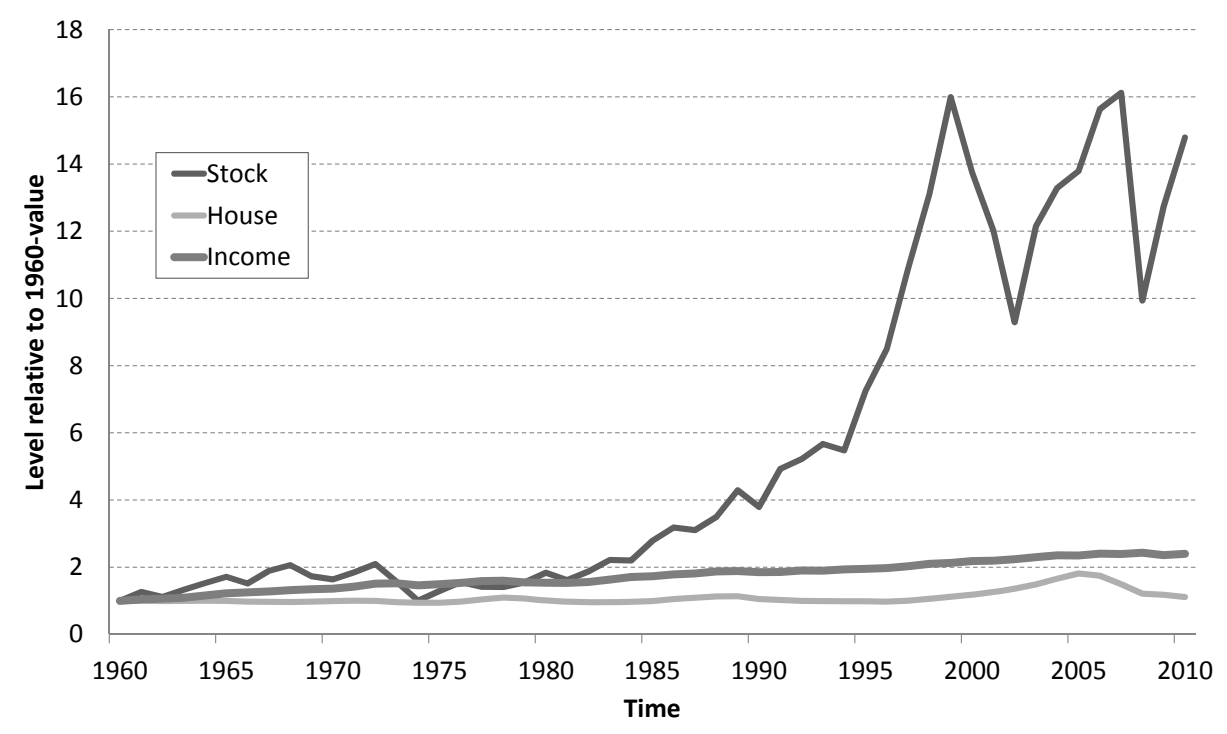

Figure 2 depicts the time series of the detrended predictors before winsorizing.

In our sample, the net payout yield is a statistically better stock predictor than various alternatives suggested in the existing literature, namely the dividend yield, the log price-earnings ratio, the cyclically adjusted log price-earnings ratio, and the GDP growth rate, and none of these predictor candidates notably improve the prediction when added along with the net payout yield. ${ }^{7}$ The net payout yield has no predictive power for income growth, and the rent-price ratio has no predictive power for stock returns.

We estimate a $\operatorname{VAR}(1)$ system which is a discretization of our continuous-time model and transform the VAR parameter estimates into estimates of our model parameters. Details are given in Appendix A. We estimate both the main model described above as well as the three special cases in which either $x$ or $y$ or both are eliminated from the system, since we are going to explore the predictors' individual and joint impact on portfolios and welfare in later sections.

Table 1 lists the parameter values used as the benchmark in the following. These values equal the empirical estimates with a few exceptions. First, we reduce the equity premium from the

\footnotetext{
${ }^{7}$ Furthermore, the GDP growth rate is not an $\mathrm{AR}(1)$ process as required in our formulations, but rather a moving average process.
} 
Figure 2: The time series of detrended predictors. The figure depicts the annual net payout yield (left panel) and the annual change of the log rent-price ratio (right panel), both series are shown before winsorizing.
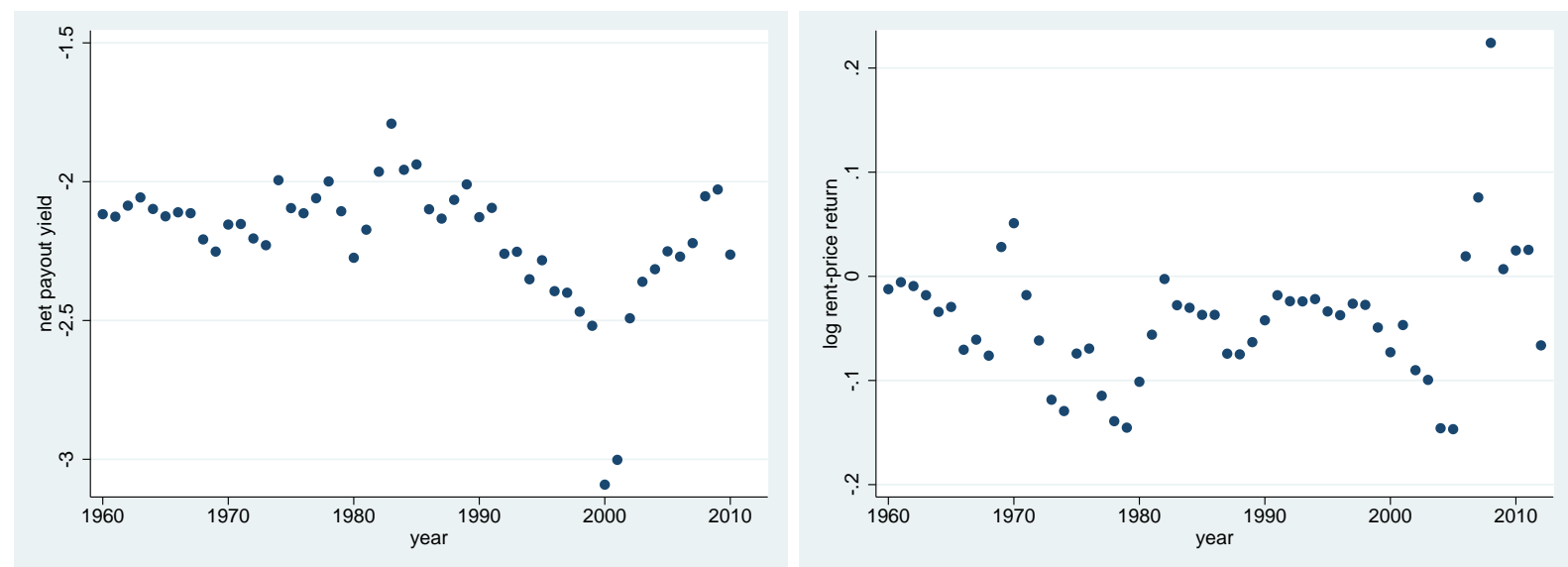

estimated $5.3 \%$ to $4 \%$ to account for survivorship bias (Brown, Goetzmann, and Ross 1995) as well as the decline in discount rates and the implied unexpected capital gains over the sample period (Fama and French 2002). Moreover, a 4\% equity premium is used in related papers such as Cocco, Gomes, and Maenhout (2005) and Yao and Zhang (2005). Secondly, the use of a house price index and aggregate income underestimates the volatilities of an individual house price and the labor income of a typical worker. We increase the volatility of house prices from the estimated value of $6.1 \%$ to $12 \%$, which is identical to the value assumed by Flavin and Yamashita (2002) and Yao and Zhang (2005) and in the range estimated by Case and Shiller (1989) and Bourassa, Haurin, Haurin, Hoesli, and Sun (2009). Furthermore, we increase the income volatility from the estimate of $2.1 \%$ to $10 \%$, in line with the estimate in Cocco, Gomes, and Maenhout (2005). Finally, the average growth rate of the aggregate income series is $1.7 \%$ per year, but this is not reflecting the income growth an individual can expect. As our benchmark we assume an expected income growth rate of $1 \%$ throughout the working life. Over the 35-year working period the income is then expected to grow by a factor $\exp (0.01 \times 35) \approx 1.42$, which seems reasonable and is close to the $38 \%$ reported as the median individual's income growth by Guvenen, Karahan, Ozkan, and Song (2015).

The net payout yield is borderline significant in predicting excess stock returns ( $p$-value of $5.1 \%$ ) and highly significant in predicting house price growth ( $p$-value of $1.0 \%)$. The positive estimate of $\chi_{S}$ shows that the net payout yield positively predicts stock prices as found by Boudoukh et al. (2007), although the slope coefficient of 0.329 is smaller than they report for the univariate regression using 1926-2003 data. The net payout yield negatively predicts house price growth as indicated by the estimate -0.106 of $\chi_{H x}$. The rent-price ratio is a significant ( $p$-value $\left.0.4 \%\right)$ negative predictor 
Table 1: Baseline parameter values. The table shows the estimates of the model parameters based on 1960-2010 US data. Here, $x$ refers to the net payout ratio and $y$ to the change in the log rent-price ratio. Some parameter estimates have been adjusted as explained in the text. (N.A. means 'not available' as the parameter is not included in that model.)

\begin{tabular}{|c|c|c|c|c|c|}
\hline \multicolumn{2}{|r|}{ Parameter } & \multicolumn{4}{|c|}{ Model specifications } \\
\hline Symbol & Explanation & Main & No $y$ & No $x$ & No $x, y$ \\
\hline \multicolumn{6}{|c|}{ Drift and volatility } \\
\hline$r$ & Interest rate & 0.010 & 0.010 & 0.010 & 0.010 \\
\hline$\mu_{S}+\bar{D}$ & Equity premium & 0.040 & 0.040 & 0.040 & 0.040 \\
\hline$\chi_{S}$ & Stock predictor coefficient & 0.329 & 0.314 & N.A. & N.A. \\
\hline$\sigma_{S}$ & Stock volatility & 0.170 & 0.170 & 0.175 & 0.171 \\
\hline$\mu_{H}$ & Excess expected house price growth & -0.010 & -0.010 & -0.010 & -0.010 \\
\hline$\chi_{H x}$ & House predictor coefficient & -0.106 & -0.148 & N.A. & N.A. \\
\hline$\chi_{H y}$ & House predictor coefficient & -0.398 & N.A. & -0.446 & N.A. \\
\hline$\sigma_{H}$ & House price volatility & 0.120 & 0.120 & 0.120 & 0.120 \\
\hline$\mu_{L}$ & Expected labor income growth & 0.010 & 0.010 & 0.010 & 0.010 \\
\hline$\chi_{L}$ & Income predictor coefficient & 0.126 & N.A. & 0.119 & N.A. \\
\hline$\sigma_{L}$ & Income volatility & 0.100 & 0.100 & 0.100 & 0.100 \\
\hline$\kappa_{x}$ & Mean reversion speed & 0.234 & 0.228 & N.A. & N.A. \\
\hline$\sigma_{x}$ & Volatility of net payout yield & 0.087 & 0.087 & N.A. & N.A. \\
\hline$\kappa_{y}$ & Mean reversion speed & 0.298 & N.A. & 0.298 & N.A. \\
\hline$\sigma_{y}$ & Volatility of rent-price ratio & 0.037 & N.A. & 0.037 & N.A. \\
\hline \multicolumn{6}{|c|}{ Correlations } \\
\hline$\rho_{H S}$ & House, stock & 0.300 & 0.302 & 0.195 & 0.210 \\
\hline$\rho_{L S}$ & Income, stock & 0.268 & 0.235 & 0.232 & 0.222 \\
\hline$\rho_{L H}$ & Income, house & 0.212 & 0.073 & 0.228 & 0.111 \\
\hline$\rho_{x S}$ & Net payout yield, stock & -0.249 & -0.248 & N.A. & N.A. \\
\hline$\rho_{y S}$ & Rent-price ratio, stock & 0.007 & N.A. & 0.028 & N.A. \\
\hline$\rho_{x H}$ & Net payout yield, house & -0.121 & -0.137 & N.A. & N.A. \\
\hline$\rho_{y H}$ & Rent-price ratio, house & -0.619 & N.A. & -0.607 & N.A. \\
\hline$\rho_{x L}$ & Net payout yield, income & -0.228 & -0.196 & N.A. & N.A. \\
\hline$\rho_{y L}$ & Rent-price ratio, income & -0.003 & N.A. & -0.003 & N.A. \\
\hline$\rho_{y x}$ & Rent-price ratio, net payout yield & -0.027 & N.A. & N.A. & N.A. \\
\hline \multicolumn{6}{|c|}{ Derived correlation parameters } \\
\hline$\hat{\rho}_{H}$ & & 0.954 & 0.953 & 0.981 & 0.978 \\
\hline$\hat{\rho}_{L H}$ & & 0.138 & 0.002 & 0.186 & 0.066 \\
\hline$\hat{\rho}_{L}$ & & 0.953 & 0.972 & 0.955 & 0.973 \\
\hline$\hat{\rho}_{x H}$ & & -0.049 & -0.065 & N.A. & N.A. \\
\hline$\hat{\rho}_{x L}$ & & -0.162 & -0.142 & N.A. & N.A. \\
\hline$\hat{\rho}_{x}$ & & 0.954 & 0.956 & N.A. & N.A. \\
\hline$\hat{\rho}_{y H}$ & & -0.651 & N.A. & -0.625 & N.A. \\
\hline$\hat{\rho}_{y L}$ & & 0.089 & N.A. & 0.112 & N.A. \\
\hline$\hat{\rho}_{y x}$ & & -0.044 & N.A. & N.A. & N.A. \\
\hline$\hat{\rho}_{y}$ & & 0.752 & N.A. & 0.772 & N.A. \\
\hline
\end{tabular}


of house price growth with a $\chi_{H y}$ coefficient of -0.398 and a significant ( $p$-value $\left.1.7 \%\right)$ positive predictor of income growth with a $\chi_{L}$ coefficient of 0.126 .

The average house price growth $r+\mu_{H}$ is estimated to be zero. The net payout yield has a mean reversion speed of 0.234 (expected half-life of $(\ln 2) / \kappa_{x} \approx 3.0$ years) and a long-run standard deviation of $\sigma_{x} / \sqrt{2 \kappa_{x}} \approx 0.127$. The rent-price ratio has a mean reversion speed of 0.298 (expected half-life of 2.3 years) and a long-run standard deviation of 0.048 .

The pairwise contemporaneous correlations between stock prices, house prices, and labor income are all positive. The stock price is positively related to the net payout yield, and since the two variables are negatively correlated, the model captures to mean reversion in stock returns. In contrast, since the house price index is both negatively related to and negatively correlated with its predictors, the model captures momentum in house prices, referred to as housing cycles by Fischer and Stamos (2013). The labor income is virtually uncorrelated with its predictor. Note that $\hat{\rho}_{L}^{2}=90.8 \%$ of the variance of income shocks is unspanned.

The correlations of prices and income with the predictors generate interesting lagged effects. For example, a positive shock to stock prices this period tends to be accompanied by a negative shock to $x$ (since $\rho_{x S}<0$ ), which increases the expected house price growth next period (since $\left.\chi_{H x}<0\right)$.

\section{The decision problem of a consumer-investor}

\subsection{Formulation of the problem}

We embed the estimated model (1)-(5) of the dynamics of stock prices $S_{t}$, house prices $H_{t}$, and labor income $L_{t}$ in the life-cycle consumption and investment choice problem of an individual agent (consumer-investor or household). We assume the individual retires at a known time $\widetilde{T}$ and lives on until a known time $T$. At retirement, the income rate drops to by a fixed proportion $1-\Upsilon$,

$$
L_{\widetilde{T}+}=\Upsilon L_{\widetilde{T}-}
$$

where $\Upsilon \in(0,1)$ is the socalled replacement rate. This is consistent with the wide-spread finalsalary pension schemes and a common assumption in the literature (e.g., Cocco et al. 2005; Lynch and Tan 2011). In our baseline case we assume $\mu_{L}(t)=\mu_{L}=0.01$ before retirement and $\mu_{L}(t)=0$ in retirement and that both the sensitivity $\chi_{L}$ towards the predictor and the income volatility are 
not changing at retirement. Hence, in contrast to most studies, we allow for retirement income risk. Even for a constant pay-out pension scheme the disposable retirement income is risky because of uncertain medical costs (see De Nardi, French, and Jones (2010)). Also, some agents continue to earn income from other sources such as proprietary businesses or other non-traded assets. Moreover, due to mortality risk, the agent may not receive future retirement pay and, while we do not formally model mortality, retirement income risk partially captures this effect parsimoniously.

The agent consumes a perishable good and housing services from living in a house (we let "house" represent any type of residential real estate). The perishable good serves as the numéraire. The agent can invest in a bank account with a constant interest rate $r$ and in the stock index with value $S_{t}$. The agent can invest in and rent houses. A house is characterized by a number of housing units, where a "unit" is a one-dimensional representation of the size, quality, and location. Prices of all houses are assumed to move in parallel. The purchase of $a$ units of housing costs $a H_{t}$. The unit rental cost of houses is assumed proportional to their market prices so that the total costs of renting $\phi$ housing units over a short period $[t, t+d t]$ are $\phi R H_{t} d t$. These assumptions are standard in the consumption and investment literature involving housing (e.g. Yao and Zhang 2005; Fischer and Stamos 2013). Following Kraft and Munk (2011) we impose no restrictions on the number of units owned and rented. In particular, simultaneous owning and renting is possible. The agent derives utility from the number of housing units occupied, whether rented or owned.

To facilitate the solution of the agent's utility maximization problem, we assume the agent can continuously adjust both the number of units rented and the number of units owned without transaction costs. Observed changes in the physical ownership of housing units seem rare and costly, but the remodeling or the extension of a house would also count as an increase in the number of housing units owned due to the higher quality or increased space. Moreover, real-life agents can also invest in housing units by purchasing shares in residential REITs (Real Estate Investment Trusts), exchange-traded funds tracking the REIT market, or other financial assets closely linked to house prices such as the Case-Shiller derivatives. ${ }^{8}$ Homeowners can thus implement short-term variations to their desired housing investment position by investing in REITs, whereas they might prefer implementing larger changes in both desired housing consumption and investment through (rare) physical transactions of housing units.

A housing investment can be seen as a physical purchase of a housing unit (by either the agent

\footnotetext{
${ }^{8}$ Well-developed REIT markets exist in many countries. Cotter and Roll (2015) study the risk and return characteristics of U.S. REITs. Tsai, Chen, and Sing (2007) report that REITs behave more and more like real estate and less and less like ordinary stocks.
} 
himself or by a REIT he holds shares in) which is then rented out. Ownership entails maintenance costs (including property taxes) equal to a constant fraction $m \geq 0$ of the property value. The rate of return on a housing investment over a period of length $d t$ is therefore

$$
(R-m) d t+\frac{d H_{t}}{H_{t}}=\left(r+\mu_{H}^{\prime}+\chi_{H x} x_{t}+\chi_{H y} y_{t}\right) d t+\sigma_{H}\left(\rho_{H S} d B_{S t}+\hat{\rho}_{H} d B_{H t}\right)
$$

where $\mu_{H}^{\prime}=\mu_{H}+R-m$ is the average excess expected return on housing investments. Let $\phi_{o t}$ and $\phi_{r t}$ denote the number of housing units owned and rented, respectively, at time $t$, and let $\phi_{f t}$ denote the housing units owned via financial assets like REITs. What matters for the agent are the total units of houses occupied, $\phi_{C t}$, which provides utility from housing services, and the total units of housing invested in, $\phi_{I t}$, either physically owned or through REITs, where

$$
\phi_{C t} \equiv \phi_{o t}+\phi_{r t}, \quad \phi_{I t} \equiv \phi_{o t}+\phi_{f t}
$$

Hence, we have a degree of freedom. Physical ownership and REITs investments complement each other, but we do not distinguish them in the model.

Let $W_{t}$ denote the tangible wealth of the agent at time $t$, which includes the positions in the bank account, the stock index, REITs, and physically owned housing units, but not the agent's human wealth, i.e., the present value of her future labor income. Let $\Pi_{S t}$ and $\Pi_{H t}=\phi_{I t} H_{t} / W_{t}$ denote the fractions of tangible wealth invested in the stock and in housing units, respectively, at time $t$. The wealth invested in the bank account is residually determined as $W_{t}\left(1-\Pi_{S t}-\Pi_{H t}\right)$. The rate of perishable consumption at time $t$ is represented by $c_{t}$. The wealth dynamics is then

$$
\begin{aligned}
d W_{t}=W_{t}[ & \left(r+\Pi_{S t}\left(\mu_{S}^{\prime}+\chi_{S} x_{t}\right)+\Pi_{H t}\left(\mu_{H}^{\prime}+\chi_{H x} x_{t}+\chi_{H y} y_{t}\right)\right) d t \\
& \left.+\left(\Pi_{S t} \sigma_{S} \Pi_{H t} \sigma_{H} \rho_{H S}\right) d B_{S t}+\Pi_{H t} \sigma_{H} \hat{\rho}_{H} d B_{H t}\right]+\left(L_{t}-c_{t}-\phi_{C t} R H_{t}\right) d t,
\end{aligned}
$$

where $\mu_{S}^{\prime}=\mu_{S}+\bar{D}$.

The objective of the investor is to maximize life-time expected utility from perishable consumption and consumption of housing services. The indirect utility function is

$$
J(t, W, H, L, x, y)=\sup _{\left(c, \phi_{C}, \Pi_{S}, \Pi_{H}\right) \in \mathcal{A}_{t}} \mathrm{E}_{t}\left[\int_{t}^{T} e^{-\delta(u-t)} U\left(c_{u}, \phi_{C u}\right) d u\right]
$$

where $W, H, L, x$, and $y$ denote time $t$ values of wealth, house price, labor income, and the two 
predictors, and where $U$ is a Cobb-Douglas-power utility function

$$
U\left(c, \phi_{C}\right)=\frac{1}{1-\gamma}\left(c^{a} \phi_{C}^{1-a}\right)^{1-\gamma}
$$

Here $\gamma>1$ is the relative risk aversion, and $a \in(0,1)$ the relative utility weight of the two goods. ${ }^{9}$ Similar preferences are assumed in other recent papers, such as Cocco (2005), Yao and Zhang (2005), and van Hemert (2010). The set $\mathcal{A}_{t}$ contains all admissible control processes over the time interval $[t, T]$. Constraints on the controls are thus reflected by $\mathcal{A}_{t}$. We shall impose the constraints

$$
\Pi_{S} \geq 0, \quad \Pi_{H} \geq 0, \quad \Pi_{S}+q \Pi_{H} \leq 1,
$$

which rule out short-selling and limits borrowing to a fraction $(1-q)$ of the current value of the housing investment. Because of incomplete markets (shocks to labor income and the predictors are not spanned by traded assets) and portfolio constraints, we are unable to solve the problem in closed form. Next, we outline our numerical solution method.

\subsection{Solution method}

We apply the SAMS (Simulation of Artificial Markets Strategies) approach introduced by Bick, Kraft, and Munk (2013) and illustrated in Figure 3. The method exploits that we can derive an explicit expression for the optimal strategy in each of various artificial markets. In any of the artificial markets the agent is unconstrained, has access to the same assets (with identical or higher returns) as in the true market plus additional assets completing the market, so the agent can obtain at least as high an expected utility as in the true market. In our case an artificial market is characterized by the adjusted Sharpe ratios on stocks and houses and Sharpe ratios on the three fictitious assets spanning the risks associated with the shocks to labor income and the two predictors. Cvitanić and Karatzas (1992) showed theoretically that the solution to the true, constrained and incomplete market problem is identical to the solution in the worst of all the artificial markets. We can solve the utility maximization problems in some artificial markets in near-closed form in the sense that the solution involves two functions determined by numerically solving standard partial differential equations. In Figure 3, the points marked to the right on the axis indicate the maximal utility in such computable artificial markets denoted by $\Theta_{1}, \Theta_{2}$, etc. The

\footnotetext{
${ }^{9}$ We disregard utility of bequests which is known to have a negligible impact on portfolio decisions except maybe in the final few years of life. In an empirical study, Hurd (1989) concludes that bequest motives in various countries are close to zero.
} 
Figure 3: The solution technique. The axis shows the agent's expected utility. "Unknown optimal" represents the indirect utility in the true market, i.e., the expected utility generated by the unknown optimal consumption-investment strategy. Each point to the right corresponds to the indirect utility in an artificial market with deterministic modifiers characterized by some parameter set $\Theta$. The corresponding strategy is transformed into a feasible strategy in the true market which generates an expected utility on the left part of the axis. The best of these strategies is derived from the optimal strategy in an artificial market for some $\Theta^{*}$. The arrows above the axis indicate the unknown utility loss and a computable upper bound on the loss the agent suffers by following the best of the considered feasible strategies instead of the unknown optimal strategy.

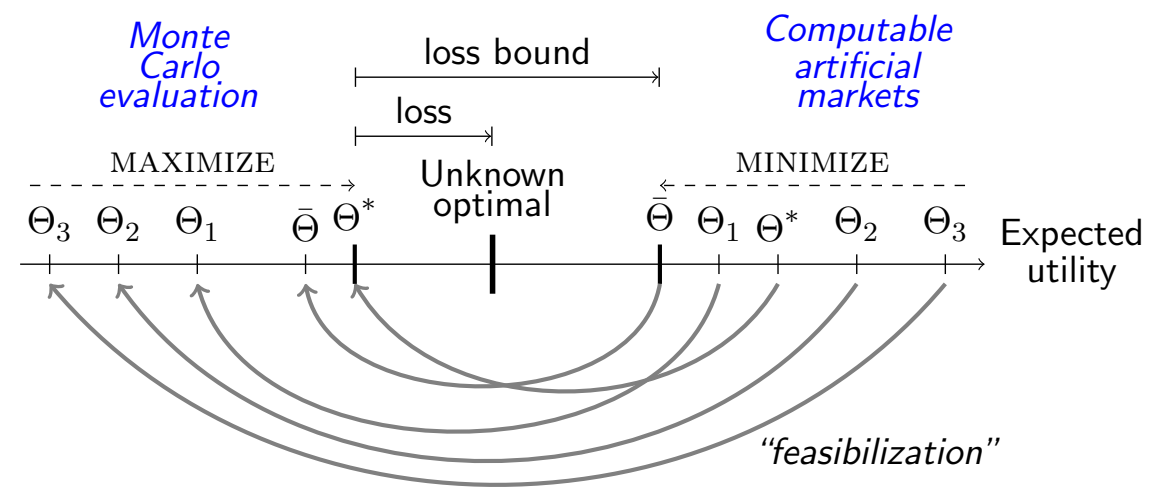

lowest expected utility among these artificial markets - indicated by $\bar{\Theta}$ on the axis - is at least as large as the unknown maximum in the true market.

In any of the computable artificial markets, the fractions of wealth optimally invested in stocks and housing units are of the form

$$
\begin{aligned}
\Pi_{S}= & \frac{1}{\gamma \hat{\rho}_{H}^{2} \sigma_{S}^{2}}\left(\mu_{S}^{\prime}(t, x, y)+\chi_{S} x-\frac{\rho_{H S} \sigma_{S}}{\sigma_{H}}\left(\mu_{H}^{\prime}(t, x, y)+\chi_{H x} x+\chi_{H y} y\right)\right) \frac{W+L F}{W} \\
& +\left(M_{x S} \frac{B_{x}}{B}+M_{y S} \frac{B_{y}}{B}\right) \frac{W+L F}{W}-\left(M_{L S}(t)+M_{x S} \frac{F_{x}}{F}+M_{y S} \frac{F_{y}}{F}\right) \frac{L F}{W}, \\
\Pi_{H}= & \frac{1}{\gamma \hat{\rho}_{H}^{2} \sigma_{H}^{2}}\left(\mu_{H}^{\prime}(t, x, y)+\chi_{H x} x+\chi_{H y} y-\frac{\rho_{H S} \sigma_{H}}{\sigma_{S}}\left(\mu_{S}^{\prime}(t, x, y)+\chi_{S} x\right)\right) \frac{W+L F}{W} \\
& +k \frac{W+L F}{W}+\left(M_{x H} \frac{B_{x}}{B}+M_{y H} \frac{B_{y}}{B}\right) \frac{W+L F}{W}-\left(M_{L H}(t)+M_{x H} \frac{F_{x}}{F}+M_{y H} \frac{F_{y}}{F}\right) \frac{L F}{W} .
\end{aligned}
$$

Here $\mu_{S}^{\prime}$ and $\mu_{H}^{\prime}$ are the adjusted expected excess stock and house returns (including dividends and rents). The functions $F=F(t, x, y)$ and $B=B(t, x, y)$ are found by solving simple partial differential equations which involve the Sharpe ratios on the fictitious assets completing the market. The product $L F$ is the human capital, i.e. the present value of income in the remaining life time, which is uniquely determined is any artificial market. The stock investment consists of the speculative demand, a term hedging the variations in expected stock returns, and an adjustment for the extent 


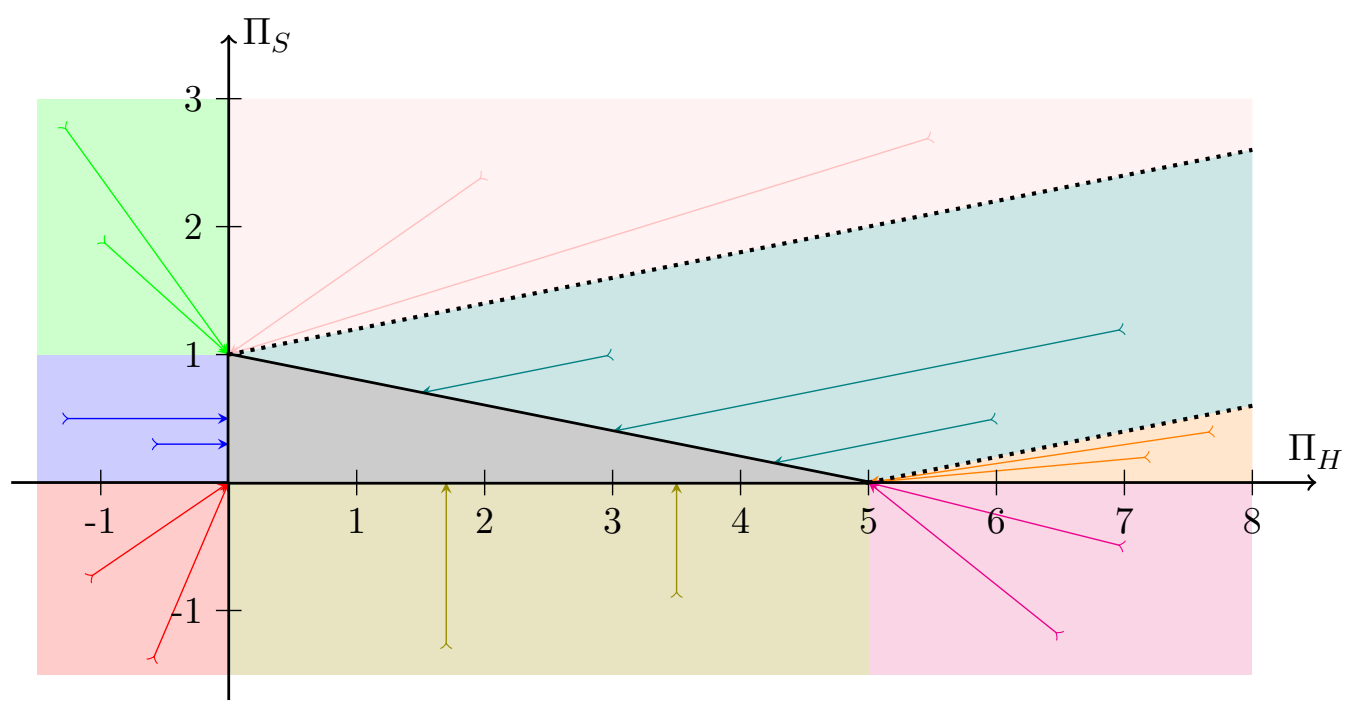

Figure 4: The transformation of portfolio weights. The gray triangle is the feasible region. The graph is drawn for $q=0.2$ so the maximal loan-to-value ratio is $80 \%$. The transformation depends on which colored area the artificial market portfolio is located in. The arrows show examples of the transformation.

to which the human capital replaces a direct stock investment. The housing investment consists of three similar terms plus the term $k \frac{W+L F}{W}$, where $k=(1-a)(\gamma-1) / \gamma$, that hedges against increases in housing consumption costs. Appendix B explains the construction of the computable artificial markets and the solution to the corresponding utility maximization problem in more detail.

The explicit, optimal strategy in any of the artificial markets is infeasible in the true market, but we can feasibilize it - that is, transform it into a feasible strategy in the true market - and then evaluate the expected utility it generates in the true market by standard Monte Carlo simulation. ${ }^{10}$ The transformation follows Cvitanić and Karatzas (1992, Ex. 14.9) and is illustrated in Figure 4. This procedure leads to the points on the left part of the axis in Figure 3. We then maximize over these feasibilized strategies and obtain the expected utility indicated by $\Theta^{*}$ in the figure. The corresponding near-optimal strategy is the strategy suggested by the SAMS approach.

Just as with other numerical methods, the suggested strategy is unlikely to be identical to the unknown, truly optimal strategy so by using the suggested strategy the agent suffers a welfare loss. By comparing the expected utility generated by the near-optimal strategy to the expected utility in

\footnotetext{
${ }^{10}$ In the artificial markets labor income is fully spanned and tangible wealth can be allowed to be temporarily negative if balanced by human capital. In the true market, tangible wealth must stay non-negative because of the unhedgeable shocks that may bring income close to zero. Follow Bick et al. (2013) we handle this by multiplying the human capital by a factor $\left(1-e^{-\eta W_{t}}\right)$, where $\eta>0$ is a constant determined experimentally. This is consistent with the intuition that future income has a smaller present value when current wealth $W_{t}$ is small. Formally we replace $F_{t}$ by $\widetilde{F}_{t}=\left(1-e^{-\eta W_{t}}\right) F\left(t, x_{t}, y_{t}\right)$.
} 
the worst of the artificial markets considered, we derive an upper bound on the welfare loss which is therefore a measure of precision of the approach. In our baseline case, the loss bound corresponds to $1.7 \%$ of the agent's wealth in certainty equivalent terms. In the examples studied by Bick et al. (2013), the true loss is significantly smaller than the loss bound. In addition to the low loss bound, the SAMS method is relatively easy to implement. As our optimization problem (8), in addition to time, features five state variables (can be reduced to four after exploiting homogeneity of the utility function), grid-based methods are infeasible with grid sizes that bring us near the continuous-time solution.

\section{Illustration and discussions of results}

This section illustrates and discusses a number of properties of the investment strategies derived using the method explained above. Unless otherwise noted, the estimation-based parameter values in Table 1 are used. Furthermore, we assume a relative risk aversion coefficient of $\gamma=5$. We set the relative utility weight of the goods to $a=0.7$, implying that total consumption expenditures consists of $70 \%$ on perishable goods and $30 \%$ on housing consumption, which seems consistent with observed household expenditure, cf. a report by the U.S. Department of Labor (2003). The subjective time preference rate is $\delta=0.05$. The agent is initially of age $t=30$, retires at age $\widetilde{T}=65$, and lives on until age $T=80$. We assume an income replacement rate of $\Upsilon=0.6$. $^{11}$ The results presented below are assuming the income growth component $\mu_{L}(t)$ is constant and equal to the estimate $\bar{\mu}_{L}$ based on aggregate income. An age-dependent income profile is included in a later version. We set the proportional rental rate to $R=0.067$ as motivated by Fischer and Stamos (2013) and assume maintenance costs of $m=0.035$ (includes property taxes that constitute 1-2\% in many U.S. states). Finally, as a benchmark we assume a $60 \%$ maximal loan-to-value ratio corresponding to $q=0.4$, but consider alternatives below.

For concreteness, we think of a housing unit as 1000 square feet of average quality and location. Using a monetary unit of a thousand U.S. dollars, we set the initial unit house price to $H=250$, which implies an initial annual rent of $\$ 12,500$ for a housing unit. Furthermore, the initial tangible wealth is set to $W=\$ 20,000$ and the initial annual income to $L=\$ 20,000$ which are roughly equal to the median values for individuals of age 30-40 in the 2007 Survey of Consumer Finances (see Kraft and Munk 2011). Finally, we set the initial values of the time-varying drift components

\footnotetext{
${ }^{11}$ The reduction from the $68 \%-93 \%$ estimate of Cocco et al. (2005) is a way to implicitly incorporate the higher medical expenses in retirement as well as the increased mortality risk that lowers expected future income.
} 
Table 2: Output grid for regression of optimal strategies. The table shows values for our state variables for which we compute the optimal strategies.

\begin{tabular}{lccc}
\hline Variable & Steps & Min. value & Max. value \\
\hline$t$ & 8 & 0 & 35 \\
$H$ & 5 & 200 & 300 \\
$L / W$ & 200 & 0.01 & 2.00 \\
$x$ & 11 & -0.85 & 0.85 \\
$y$ & 11 & -0.25 & 0.25 \\
\hline
\end{tabular}

of house prices and income to $x=y=0$.

\subsection{Optimal decisions}

The optimal strategies are generally depending on time and five state variables, and are thus difficult to depict graphically in an accessible way. To get an impression of the determinants of the optimal strategies, we perform a linear regression of $\pi_{S}, \pi_{H}$, and $c / W$ on the state variables and some interaction terms. ${ }^{12}$ For tractability the regression is based on the optimal strategies for the selected values for our state variables shown in Table 2. The regression output is shown in Table 3. The very high $R^{2}$ values indicate that the linear relation well approximates the true relation between the decision variables and the explanatory variables. The stock weight tends to be increasing in age, in the stock return predictor $x$ (a well-known result since $x$ generates mean reversion in stock returns), and in the house and income predictor $y$. The stock weight is decreasing in the labor-wealth ratio. Conversely, the housing weight tends to be decreasing in age, in the stock market predictor, and in the house and income predictor. The latter is due to the fact that $y$ is generating momentum in house prices, not mean reversion.

To assess life-cycle patterns, we perform the following analysis. Based on our near-optimal life-cycle consumption, housing, and investment strategy, we simulate 100,000 paths of exogenous state variables and wealth (applying this strategy) forward, and in the figures mentioned below we report expectations of consumption, wealth, investments, and portfolio weights computed by averaging over the simulations. ${ }^{13}$

Figure 5 illustrates the optimal investment strategy over the life cycle with baseline parameter

\footnotetext{
${ }^{12}$ Since the optimal spending on housing consumption relative to perishable consumption is equal to a constant we do not regress $\phi_{C}$ separately.

${ }^{13}$ We simulate using Euler discretizations with monthly time steps. Note that $F$ and $B$ involve numerical integration. Before running the simulations, we evaluate $F$ and $B$ on grids, and when we need values for $F$ and $B$ and their derivatives in the simulations, we use the grid values and linear interpolation.
} 
Table 3: Regression results for optimal strategies. The table shows the regression results based on the state variable values shown in Table 2. All parameters are significant at the $0.1 \%$ level.

\begin{tabular}{lccc}
\hline & $\pi_{S}$ & $\pi_{H}$ & $c / W$ \\
\hline$t$ & 0.004 & -0.004 & 0.000 \\
$x$ & 1.615 & -1.839 & 0.002 \\
$y$ & 1.116 & -5.400 & 0.005 \\
$L / W$ & -0.703 & 7.466 & 0.646 \\
$t \times L / W$ & 0.055 & -0.110 & -0.000 \\
$x \times L / W$ & 20.269 & -23.866 & -0.068 \\
$y \times L / W$ & 14.050 & -66.331 & 0.219 \\
Constant & 0.170 & 0.545 & 0.043 \\
$R^{2}$ & 0.980 & 0.982 & 0.931 \\
Obs. & 968,000 & 968,000 & 968,000 \\
\hline
\end{tabular}

values. The horizontal axes show time passed after the initial date where the agent is assumed to be of age 30. The left panel shows the amounts invested in the housing asset, the stock index, and the risk-free asset (bond), whereas the right panel depicts the portfolio weights relative to tangible wealth. The left panel shows that the agent builds up wealth in the active phase to finance consumption in retirement where income is markedly lower. The portfolio is dominated by housing, especially early in life, where the investment is fully leveraged. Later in life, borrowing is less than the allowed $60 \%$ of the house value. The stock weight is around $25 \%$ early in life, but increases rather quickly to around 50\%, where it remains relatively stable. In settings ignoring housing, labor income typically leads to a full stock investment being optimal (leveraged if possible), cf. Cocco et al. (2005), even for relatively high levels of risk aversion. Our results confirm the findings of Cocco (2005), among others, that housing crowds out stocks. Not only is housing a decent investment in itself (especially considering the rents), it also provides access to leverage, and constitutes a hedge against increases in housing consumption costs.

The role of leverage is clear from Figure 6 which compares the portfolio weights for $q \in$ $\{0.2,0.4,1.0\}$, corresponding to a maximal loan-to-value ratio of $80 \%, 60 \%$, or $0 \%$, respectively. As an investment, the house is not nearly as attractive early in life if it does not give access to leverage in which case the stock has a larger weight in the portfolio.

\subsection{The effect of predictability}

Now, we compare the investment profiles of an agent accounting for predictability and an agent disregarding predictability. The latter agent assumes that the dynamics of stock prices, house prices, 
Figure 5: Optimal investments over the life cycle: baseline case. The left panel shows the expected tangible wealth and its decomposition into stock investment, housing investment, and risk-free (bond) investment (the unit is thousands of U.S. dollars). The right panel shows the expected percentage investments of tangible wealth into stocks, housing, and bonds. Baseline parameter values are used.
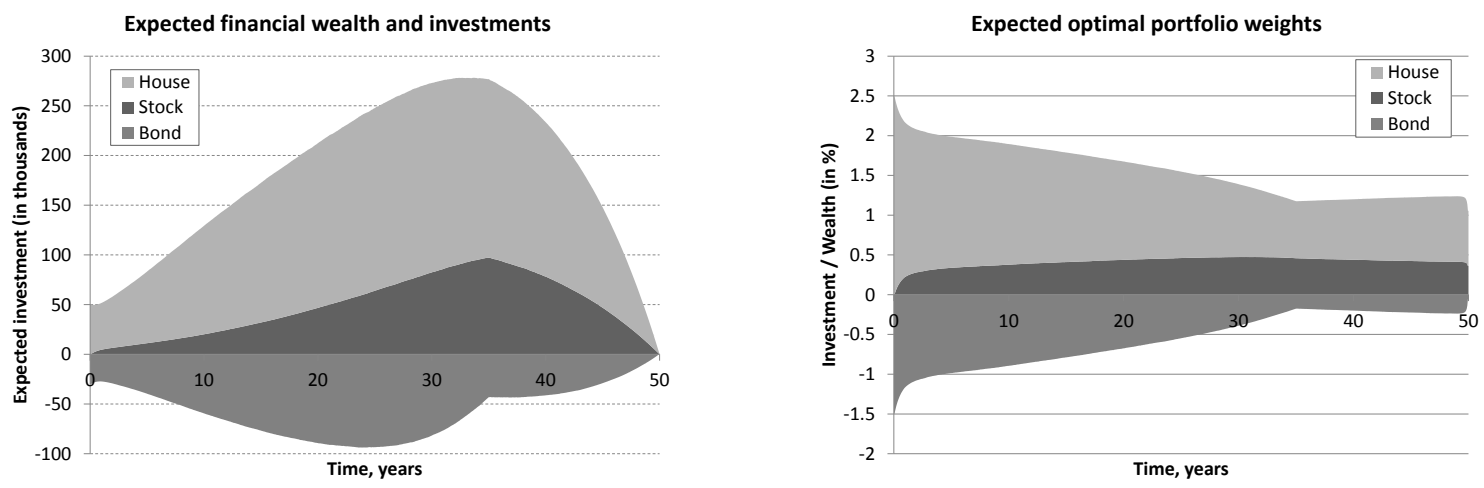

Figure 6: The effect of collateralized borrowing on portfolio weights. The left [right] panel shows the expected percentage investments of financial wealth in stocks [housing assets] for three different values of the housing collateral parameter $q$. Baseline values are used for other parameters.
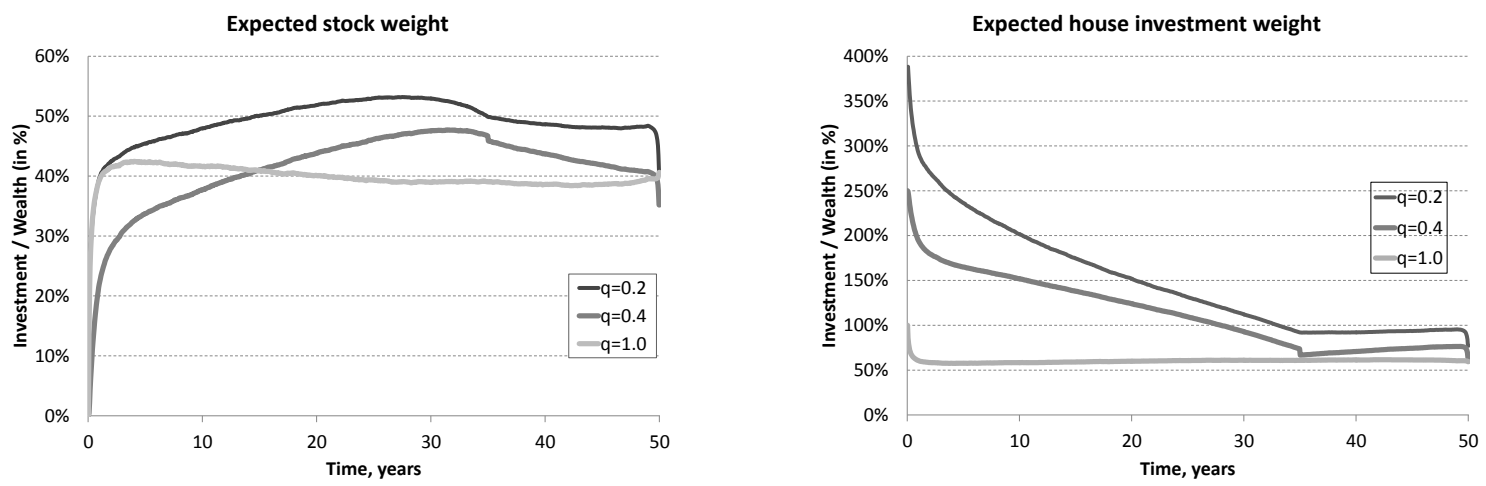
Figure 7: The effect of predictability on investments. The left [right] panel shows the expected percentage investments of tangible wealth in stocks [housing assets]. Baseline parameter values are used.
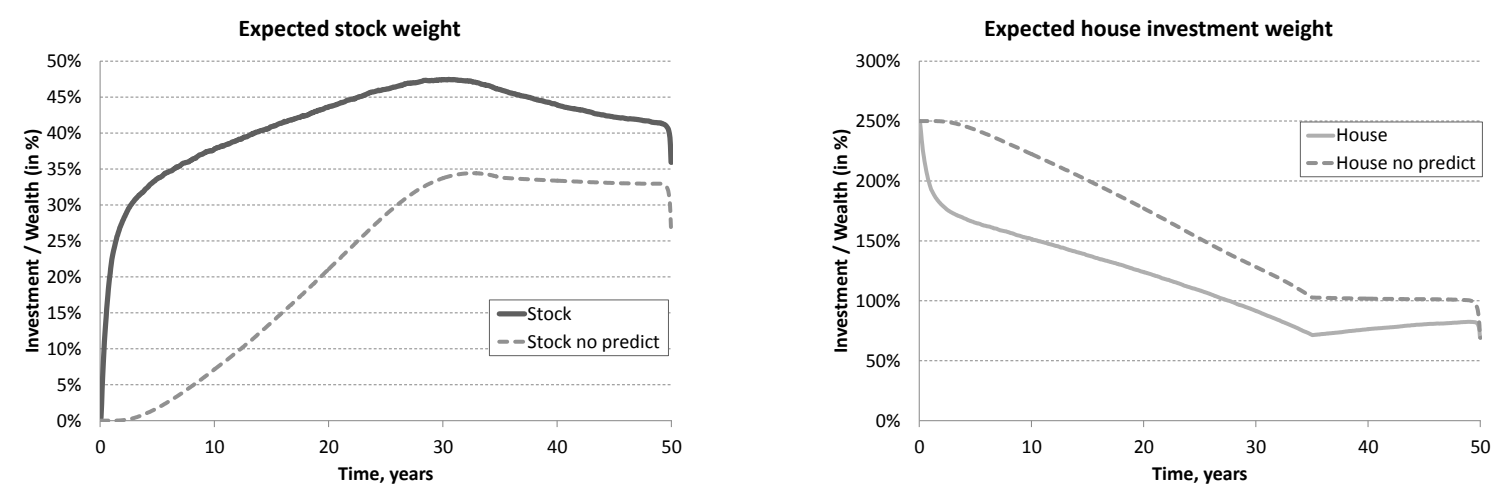

and labor income are given by (1)-(3), but without the terms involving $x$ and $y$, i.e., effectively imposing $\chi_{S}=\chi_{H x}=\chi_{H y}=\bar{\chi}_{L}(t)=0$, and thus arriving at the parameter estimates in the right-most column of Table 1 . The two agents' expected portfolio weights over the life cycle are shown in Figure 7. Predictability materializes as mean reversion in stock returns and thus leads to a larger average portfolio share of the stock as found in simpler settings by Kim and Omberg (1996) and others. In contrast, predictability in house price growth emerges as momentum and therefore lowers the average share of housing in the portfolio.

By taking predictability into account, the agent can better time the market and thus generate higher investment returns, which leads to a higher average consumption level. The top panel of Table 4 reports the present value of the difference in future consumption levels between the case in which predictability is accounted for and the case in which it is not. ${ }^{14}$ The present value is computed by discounting the average consumption difference each year by the risk-free rate of $1 \%$. The present value of consumption differences over the entire 50 -year adult period is $\$ 179,137.63$, which is sizable compared to the assumed initial financial wealth and initial annual income that both are $\$ 20,000$. The present value of consumption differences until retirement is $\$ 74,394.99$, so the present value difference of $\$ 104,742.75$ summarizes the effect on retirement consumption. Ignoring predictability in stock returns, house prices, and labor income leads to lower returns on investments, lower savings, and thus significantly lower consumption in retirement. Strategies based on predictability not only leads to different levels of consumption, but also reduces the

\footnotetext{
${ }^{14}$ The relative utility weight of the two types of consumption is $a=0.7$, so $70 \%$ of the consumption differences are attributed to perishable consumption and $30 \%$ to housing consumption.
} 
Table 4: Welfare effect of predictability. PV is the present value at time $t_{0}$ of the difference between the optimal consumption with or without predictability. The present value is calculated by discounting the average consumption difference at a $1 \%$ rate. RWEL is the certainty equivalent of the consumption difference measured as a percent of initial total wealth. Baseline parameter values are used.

\begin{tabular}{|c|c|c|c|c|}
\hline & \multicolumn{2}{|c|}{ Until death } & \multicolumn{2}{|c|}{ Until retirement } \\
\hline & $\mathrm{PV}$ & RWEL & $\mathrm{PV}$ & RWEL \\
\hline All paths & $179,137.63$ & $5.57 \%$ & $74,394.88$ & $4.30 \%$ \\
\hline \multicolumn{5}{|c|}{ Sorted on $x$ outcomes } \\
\hline $25 \%$ upper & $174,413.73$ & $7.38 \%$ & $71,388.70$ & $5.57 \%$ \\
\hline $50 \%$ upper & $165,637.71$ & $6.53 \%$ & $69,017.44$ & $4.95 \%$ \\
\hline $50 \%$ lower & $191,450.59$ & $4.39 \%$ & $79,971.43$ & $3.56 \%$ \\
\hline $25 \%$ lower & $216,569.51$ & $4.14 \%$ & $89,580.24$ & $3.36 \%$ \\
\hline \multicolumn{5}{|c|}{ Sorted on $x$ volatility } \\
\hline $25 \%$ upper & $228,253.10$ & $6.74 \%$ & $90,631.17$ & $5.21 \%$ \\
\hline $50 \%$ upper & $207,432.06$ & $6.20 \%$ & $84,058.60$ & $4.79 \%$ \\
\hline $50 \%$ lower & $150,843.19$ & $4.95 \%$ & $64,731.17$ & $3.81 \%$ \\
\hline $25 \%$ lower & $139,580.23$ & $4.59 \%$ & $60,525.53$ & $3.58 \%$ \\
\hline \multicolumn{5}{|c|}{ Sorted on y outcomes } \\
\hline $25 \%$ upper & $118,610.91$ & $6.17 \%$ & $55,833.61$ & $5.07 \%$ \\
\hline $50 \%$ upper & $123,763.50$ & $5.90 \%$ & $56,733.31$ & $4.66 \%$ \\
\hline $50 \%$ lower & $233,324.79$ & $5.31 \%$ & $92,255.55$ & $4.04 \%$ \\
\hline $25 \%$ lower & $307,655.46$ & $5.49 \%$ & $116,817.37$ & $4.13 \%$ \\
\hline \multicolumn{5}{|c|}{ Sorted on y volatility } \\
\hline $25 \%$ upper & $269,948.74$ & $6.96 \%$ & $107,529.07$ & $5.20 \%$ \\
\hline $50 \%$ upper & $225,573.71$ & $6.32 \%$ & $91,302.29$ & $4.73 \%$ \\
\hline $50 \%$ lower & $132,701.55$ & $4.87 \%$ & $57,487.47$ & $3.90 \%$ \\
\hline $25 \%$ lower & $114,883.42$ & $4.35 \%$ & $50,976.32$ & $3.56 \%$ \\
\hline
\end{tabular}

likelihood of ending up in states with low consumption and thus very low utility. We capture this by the certainty equivalent of wealth. The consumption differences over the entire 50-year adult life span correspond to a certainty equivalent of wealth of $5.57 \%$, which can be interpreted as if an agent ignoring predictability is giving up $5.57 \%$ of total initial wealth. Here total initial wealth is computed as the sum of initial financial wealth and the present value of future income (average income discounted at $1 \%)$.

Table 4 also dissects the welfare effects of predictability by considering various subsamples of paths. Along each sample path, we compute the average and standard deviation of the two predictors. First, we sort the sample paths based on the pathwise average value of the net payout yield $x$. In the subsample consisting of the $25 \%$ of the paths with the highest average $x$, we find that the present value of consumption differences is $\$ 174,413.73$ and that the certainty equivalent of 
consumption differences corresponds to $7.38 \%$ of total initial wealth. Compared to the full sample, the average effect of predictability on the consumption level is slightly smaller in this subsample, but predictability is very effective in eliminating states with very low consumption. Conversely, in the subsample of the $25 \%$ of the paths with the lowest average $x$, predictability has a larger effect on the average consumption level, but a smaller (although still sizeable) effect on expected utility. When we sort on average outcomes of the log rent-price ratio $y$, we find that incorporating the predictability represented by $y$ has the largest effect on average consumption when $y$ tends to be low, but the largest effect on expected utility when $y$ tends to be high. When sorting on the volatility of $x$ or $y$, we see that it is particularly important to base the strategy on predictability when the predictors are very volatile. In this case, expected stock and house returns and expected income growth vary more so market timing is more important for increasing average returns and for avoiding low consumption states.

Table 5 repeats the above analysis for the no borrowing case, $q=1$. If the agent cannot engage in collateralized borrowing, she cannot invests as aggressively in stocks and housing in the situations where the predictors would induce that behavior and, consequently, the agent builds up less wealth and consumes at a lower level. In particular, when the log rent-price ratio $y$ is low, an unconstrained agent would invest a lot in housing, but this would often violate a strict borrowing constraint. Therefore, in the no borrowing case, predictability has a smaller effect on expected utility and, in particular, on the average consumption level.

\subsection{Historical simulations}

Figure 2 shows the realized path of the two predictors over our sample period 1960-2010. How important was it for investors living in this period to take predictability into account? With our timing convention, an agent entering our model at age 30 in 1961, retired in 1996, and passed away in 2011, so her life span is exactly covered by our sample period. Of course, along a given sample path including the realized path, the strategy derived assuming predictability is not guaranteed to perform better than the strategy ignoring predictability. However, as shown in Table 6, the agent taking predictability into account would generally have consumed at a significantly higher level with the present value (in 1961) of consumption differences equalling $\$ 44,168.56$ until the retirement date. Furthermore, the predictability-based strategy would have generated a higher terminal wealth with a present value of $\$ 19,779.72$. The total effect thus corresponds to $\$ 63,948.28$.

Table 6 further considers agents entering our model in the years 1962 to 1976 whom we can all 
Table 5: Welfare effect of predictability: the no-borrowing case. PV is the present value at time $t_{0}$ of the difference between the optimal consumption with or without predictability. The present value is calculated by discounting the average consumption difference at a $1 \%$ rate. RWEL is the certainty equivalent of the consumption difference measured as a percent of initial total wealth. Baseline parameter values are used except that $q=1$ so that no borrowing is possible.

\begin{tabular}{|c|c|c|c|c|}
\hline & \multicolumn{2}{|c|}{ Until death } & \multicolumn{2}{|c|}{ Until retirement } \\
\hline & PV & RWEL & PV & RWEL \\
\hline All paths & $120,927.75$ & $5.00 \%$ & $48,257.01$ & $3.80 \%$ \\
\hline \multicolumn{5}{|c|}{ Sorted on $x$ outcomes } \\
\hline $25 \%$ upper & $177,173.94$ & $7.11 \%$ & $70,639.08$ & $5.39 \%$ \\
\hline $50 \%$ upper & $150,100.85$ & $6.29 \%$ & $60,494.49$ & $4.72 \%$ \\
\hline $50 \%$ lower & $87,629.29$ & $3.72 \%$ & $34,445.81$ & $2.78 \%$ \\
\hline $25 \%$ lower & $75,778.83$ & $3.36 \%$ & $28,869.68$ & $2.48 \%$ \\
\hline \multicolumn{5}{|c|}{ Sorted on $x$ volatility } \\
\hline $25 \%$ upper & $150,579.93$ & $6.01 \%$ & $57,000.58$ & $4.52 \%$ \\
\hline $50 \%$ upper & $137,031.59$ & $5.50 \%$ & $52,864.59$ & $4.16 \%$ \\
\hline $50 \%$ lower & $104,824.02$ & $4.47 \%$ & $43,649.40$ & $3.43 \%$ \\
\hline $25 \%$ lower & $97,830.71$ & $4.50 \%$ & $41,419.56$ & $3.34 \%$ \\
\hline \multicolumn{5}{|c|}{ Sorted on y outcomes } \\
\hline $25 \%$ upper & $111,386.76$ & $5.40 \%$ & $51,892.21$ & $4.52 \%$ \\
\hline $50 \%$ upper & $112,154.29$ & $5.35 \%$ & $50,057.93$ & $4.25 \%$ \\
\hline $50 \%$ lower & $125,575.84$ & $4.92 \%$ & $44,882.38$ & $3.50 \%$ \\
\hline $25 \%$ lower & $134,809.26$ & $4.90 \%$ & $44,265.04$ & $3.37 \%$ \\
\hline \multicolumn{5}{|c|}{ Sorted on y volatility } \\
\hline $25 \%$ upper & $131,873.76$ & $4.99 \%$ & $51,190.98$ & $3.70 \%$ \\
\hline $50 \%$ upper & $125,549.17$ & $4.80 \%$ & $49,534.80$ & $3.66 \%$ \\
\hline $50 \%$ lower & $116,306.43$ & $5.20 \%$ & $46,979.18$ & $3.95 \%$ \\
\hline $25 \%$ lower & $114,527.72$ & $4.92 \%$ & $46,612.73$ & $3.79 \%$ \\
\hline
\end{tabular}


Table 6: Welfare effect of predictability along realized path. We calculate the value of including predictors in consumption and investment strategies along the observed sample path in 19602010. $P V$ cons diff is the present value at time $t_{0}$ of the difference between the pre-retirement consumption levels with and without predictability. $P V W$ diff is the present value at time $t_{0}$ of the difference between the financial wealth at retirement date. Net effect is the sum of the total consumption difference $P V$ cons diff and the retirement wealth difference $P V W$ diff. Baseline parameter values are used.

\begin{tabular}{cccc}
\hline start & PV cons diff & PV W diff & Net effect \\
\hline \multicolumn{4}{c}{ Only active phase } \\
1961 & $44,168.56$ & $19,779.72$ & $63,948.28$ \\
1962 & $42,424.15$ & $13,599.56$ & $56,023.71$ \\
1963 & $41,226.30$ & $1,270.96$ & $42,497.26$ \\
1964 & $36,539.25$ & $-8,208.78$ & $28,330.47$ \\
1965 & $35,294.67$ & $-12,174.76$ & $23,119.90$ \\
1966 & $37,928.28$ & $4,387.58$ & $42,315.86$ \\
1967 & $40,176.31$ & $16,168.53$ & $56,344.84$ \\
1968 & $42,407.70$ & $35,607.30$ & $78,015.00$ \\
1969 & $44,961.80$ & $41,865.70$ & $86,827.50$ \\
1970 & $48,537.36$ & $54,020.10$ & $102,557.46$ \\
1971 & $52,625.47$ & $66,891.00$ & $119,516.47$ \\
1972 & $58,078.04$ & $51,935.24$ & $110,013.28$ \\
1973 & $60,738.29$ & $61,498.17$ & $122,236.45$ \\
1974 & $68,406.69$ & $28,164.25$ & $96,570.94$ \\
1975 & $74,097.57$ & $47,252.88$ & $121,350.45$ \\
1976 & $71,407.03$ & $59,083.07$ & $130,490.10$ \\
\hline
\end{tabular}

follow until retirement 35 years later. All cohorts generated larger consumption levels by conditioning their decisions on the predictors and most generated larger wealth at retirement, especially the 1970-1976 cohorts since one or both predictors have been sizeable in most years since 1970 . The 1964-1965 cohorts generated lower wealth at retirement by including the predictors, but this is more than outweighed by increased consumption levels. Figure 8 illustrates the effects for different cohorts graphically.

\section{Conclusion}

We have estimated a model of the joint dynamics of stock prices, house prices, and labor income in the United States over the period 1960-2010. We have shown that the net corporate payout yield positively predicts stock house prices and negatively predicts house prices, whereas the log home rent-price ratio negatively predicts house price growth and positively predicts labor income growth.

When embedding the estimated dynamics into a rich consumption and investment choice model, we find that life-cycle investors can benefit significantly from conditioning their decisions on the two 
Figure 8: The dollar value of including predictability along the observed sample path. We calculate the value of including predictors in consumption and investment strategies along the observed sample path in 1960-2010. PV cons diff is the present value at time $t_{0}$ of the difference between the pre-retirement consumption levels with and without predictability. $P V W$ diff is the present value at time $t_{0}$ of the difference between the financial wealth at retirement date. Net effect is the sum of the total consumption difference $P V$ cons diff and the retirement wealth difference $P V W$ diff. Baseline parameter values are used.

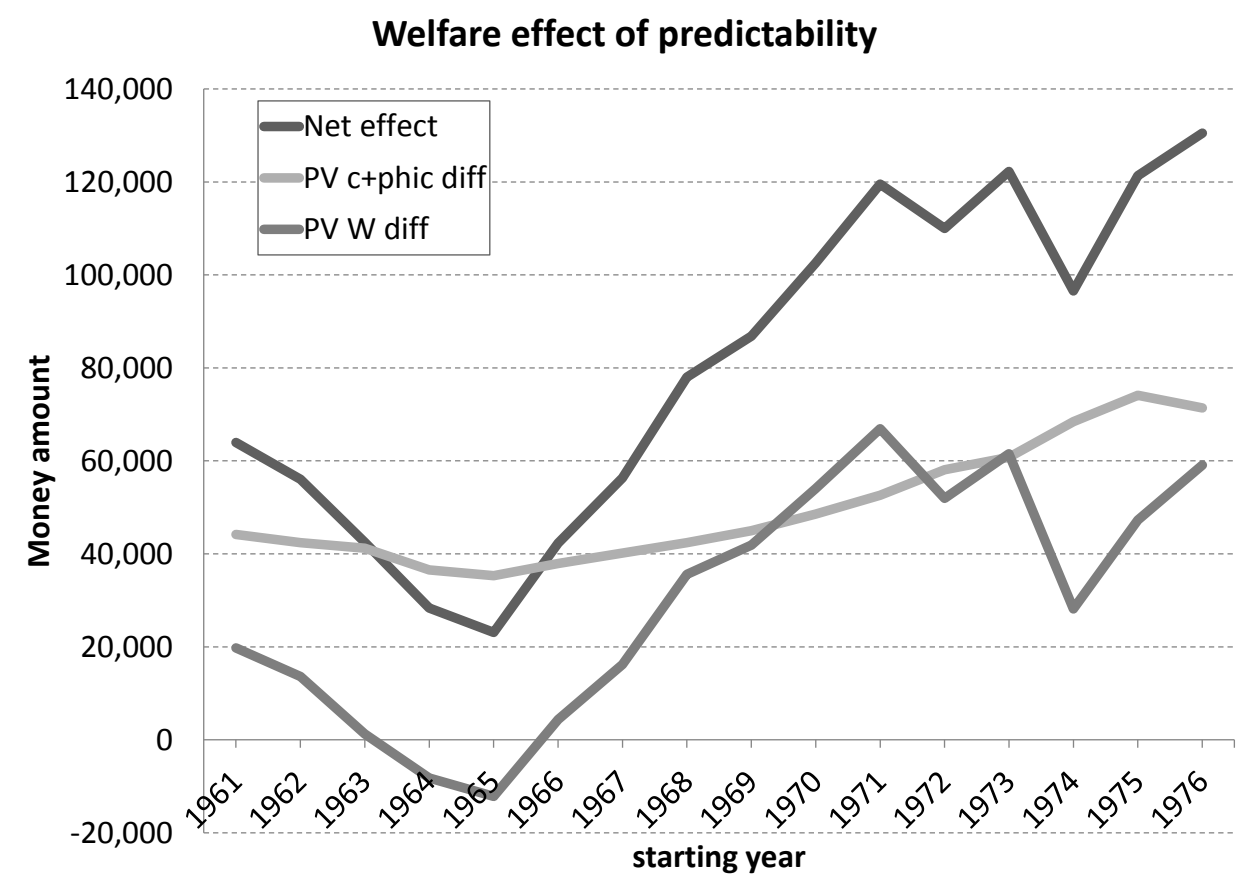


predictors. By doing so, the investors generate higher returns and thus higher average consumption, and investors can also reduce the likelihood of ending up in states with very low consumption and thus very low utility. In the baseline case, the benefits can be quantified as a present value of around $\$ 179,000$ or $5.6 \%$ of initial wealth in certainty equivalent terms. In an innovative scenariobased analysis, we show that in certainty-equivalent terms, the benefits of predictor-conditioning strategies are largest when the net payout yield and the rent-price ratio are large and volatile. Furthermore, we show that all cohorts of 30-year olds entering our model in each of the years 1961 to 1976 would have benefited from implementing conditional strategies, in particular the later of these cohorts. 


\section{A Details of the calibration}

We estimate the following $\operatorname{VAR}(1)$

$$
\left(\begin{array}{c}
r_{S, t+1}-r_{t+1} \\
r_{H, t+1}-r_{t+1} \\
r_{L, t+1} \\
x_{t+1} \\
y_{t+1}
\end{array}\right)=\left(\begin{array}{c}
\beta_{S} \\
\beta_{H} \\
\beta_{L} \\
\beta_{x} \\
\beta_{y}
\end{array}\right)+\left(\begin{array}{cc}
\beta_{x, S} & \beta_{y, S} \\
\beta_{x, H} & \beta_{y, H} \\
\beta_{x, L} & \beta_{y, L} \\
\beta_{x, x} & 0 \\
0 & \beta_{y, y}
\end{array}\right)\left(\begin{array}{c}
x_{t} \\
y_{t}
\end{array}\right)+\left(\begin{array}{c}
\varepsilon_{S, t+1} \\
\varepsilon_{H, t+1} \\
\varepsilon_{L, t+1} \\
\varepsilon_{x, t+1} \\
\varepsilon_{y, t+1}
\end{array}\right)
$$

where $\left(\varepsilon_{S}, \varepsilon_{H}, \varepsilon_{L}, \varepsilon_{x}, \varepsilon_{y}, \varepsilon_{z}\right) \sim N(0, \Sigma)$. The Stata regression output is shown in Table 7 .

At first the full model SxyHxyLxy is calibrated with change of the log rent-price ratio and net payout ratio as predictor for the stock return, house return and labor income return. In a next step, we exclude all non-significant predictors and arrive at the model SxHxyLy. In this model all predictability coefficients are at least significant at the $5 \%$ significance level. This is our benchmark model. Additionally, we report the estimation results for our sub-models: $S x H x L$ we have only $x$ as predictor, $S H y L y$ we have only $y$ as predictor and $S H L$ we ignore predictability. Table 1 reports the resulting parameter values for the different model specifications with some estimates adjusted as explained in the main text.

\section{B Details on the numerical method}

\section{B.1 Artificial markets}

Building on the idea of Cvitanić and Karatzas (1992), the constrained, incomplete market problem is embedded in a family of artificial, unconstrained, complete market problems for which we can derive exact closed-form solutions. In order to handle the constraints (10), we modify the risk-free rate as well as the drift rates of the stock and the house as follows

$$
\mu_{S t}=\mu_{S}+\nu_{S t}, \quad \mu_{H t}=\mu_{H}+\nu_{H t}, \quad r_{t}=r+\max \left(\nu_{S t}^{-}, \frac{1}{q} \nu_{H t}^{-}\right)
$$

where $\nu^{-}=\max (-\nu, 0)$; see Cvitanić and Karatzas (1992) and Bick, Kraft, and Munk (2013, Sec. 8). Note that for any values of $\nu_{S t}$ and $\nu_{H t}$, we have $r_{t} \geq r$ and $r_{t}+\mu_{S t} \geq r+\mu_{S}$ as well as $r_{t}+\mu_{H t} \geq r+\mu_{H}$. Intuitively, if the unconstrained $\Pi_{S}$ or $\Pi_{H}$ is above 1 , we increase the risk-free rate to make investing in the bank account relatively more attractive and bring down the risky investment. Conversely, if the unconstrained $\Pi_{S}$ or $\Pi_{H}$ is negative, we increase the drift rate to boost the investment in the asset. To complete the market in our case, we introduce an artificial asset for the idiosyncratic labor income risk, for 
Table 7: Regression results for model with cum returns for the stock. The table shows the regression results based on annual data from 1960 to 2010. The house market uses the national Case/Shiller home price index, for stock market data we use returns on the CRSP value-weighted market portfolio inclusive of the NYSE, AMEX, and NASDAQ markets. The risk-free asset is the Treasury bill yield from the Risk Free File on CRSP Bond tape. From NIPA tables, we obtain U.S. data for aggregated disposable personal income (per capita). To obtain real values, all time-series are deflated using the consumer price index (CPI) taken from CRSP. For the predictor variables we use for $x$ the net payout ratio, and for $y$ the change of the log rent-price ratio. In parentheses are the p-values. ${ }^{*} p<0.05$, ${ }^{* *} p<0.01,{ }^{* * *} p<0.001$

\begin{tabular}{|c|c|c|c|c|c|}
\hline & SxyHxyLxy & SxHxyLy & SxHxL & SHyLy & SHL \\
\hline \multicolumn{6}{|l|}{ Exc_ret_stock } \\
\hline Lagged_x & $\begin{array}{c}0.300 \\
(0.085)\end{array}$ & $\begin{array}{c}0.329 \\
(0.051)\end{array}$ & $\begin{array}{c}0.314 \\
(0.063)\end{array}$ & & \\
\hline Lagged_y & $\begin{array}{l}-0.164 \\
(0.719)\end{array}$ & & & & \\
\hline Constant & $\begin{array}{c}0.041 \\
(0.090)\end{array}$ & $\begin{array}{c}0.041 \\
(0.088)\end{array}$ & $\begin{array}{c}0.041 \\
(0.088)\end{array}$ & $\begin{array}{c}0.041 \\
(0.100)\end{array}$ & $\begin{array}{c}0.041 \\
(0.100)\end{array}$ \\
\hline \multicolumn{6}{|l|}{ Exc_ret_house } \\
\hline Lagged_x & $\begin{array}{c}-0.114^{* *} \\
(0.007)\end{array}$ & $\begin{array}{c}-0.106^{* *} \\
(0.010)\end{array}$ & $\begin{array}{c}-0.148^{*} \\
(0.010)\end{array}$ & & \\
\hline Lagged_y & $\begin{array}{c}-0.412^{* *} \\
(0.004)\end{array}$ & $\begin{array}{c}-0.398^{* *} \\
(0.004)\end{array}$ & & $\begin{array}{c}-0.446^{* *} \\
(0.003)\end{array}$ & \\
\hline Constant & $\begin{array}{l}-0.012 \\
(0.110)\end{array}$ & $\begin{array}{l}-0.012 \\
(0.112)\end{array}$ & $\begin{array}{l}-0.011 \\
(0.162)\end{array}$ & $\begin{array}{c}-0.012 \\
(0.133)\end{array}$ & $\begin{array}{l}-0.011 \\
(0.195)\end{array}$ \\
\hline \multicolumn{6}{|l|}{ Ret_income } \\
\hline Lagged_x & $\begin{array}{l}-0.016 \\
(0.443)\end{array}$ & & & & \\
\hline Lagged_y & $\begin{array}{c}0.126^{*} \\
(0.020)\end{array}$ & $\begin{array}{c}0.126^{*} \\
(0.017)\end{array}$ & & $\begin{array}{l}0.119^{*} \\
(0.028)\end{array}$ & \\
\hline Constant & $\begin{array}{c}0.018^{* * *} \\
(0.000)\end{array}$ & $\begin{array}{c}0.018^{* * *} \\
(0.000)\end{array}$ & $\begin{array}{c}0.017^{* * *} \\
(0.000)\end{array}$ & $\begin{array}{c}0.018^{* * *} \\
(0.000)\end{array}$ & $\begin{array}{c}0.017^{* * *} \\
(0.000)\end{array}$ \\
\hline \multicolumn{6}{|l|}{$\mathrm{x}$} \\
\hline Lagged_x & $\begin{array}{c}0.782^{* * *} \\
(0.000)\end{array}$ & $\begin{array}{c}0.766^{* * * *} \\
(0.000)\end{array}$ & $\begin{array}{c}0.772^{* * *} \\
(0.000)\end{array}$ & & \\
\hline Constant & $\begin{array}{c}0.002 \\
(0.879)\end{array}$ & $\begin{array}{c}0.002 \\
(0.880)\end{array}$ & $\begin{array}{c}0.002 \\
(0.879)\end{array}$ & & \\
\hline \multicolumn{6}{|l|}{$\mathbf{y}$} \\
\hline Lagged_y & $\begin{array}{c}0.702^{* * *} \\
(0.000)\end{array}$ & $\begin{array}{c}0.702^{* * * *} \\
(0.000)\end{array}$ & & $\begin{array}{c}0.702^{* * *} \\
(0.000)\end{array}$ & \\
\hline Constant & $\begin{array}{l}-0.000 \\
(0.973)\end{array}$ & $\begin{array}{l}-0.000 \\
(0.973)\end{array}$ & & $\begin{array}{l}-0.000 \\
(0.972)\end{array}$ & \\
\hline Obs. & 50.000 & 50.000 & 50.000 & 50.000 & 50.000 \\
\hline
\end{tabular}


the $x$ risk, and for the $y$ risk with price processes given by

$$
\begin{aligned}
& d V_{x t}=V_{x t}\left[\left(r_{t}+\lambda_{x t}\right) d t+d B_{x t}\right], \\
& d V_{y t}=V_{y t}\left[\left(r_{t}+\lambda_{y t}\right) d t+d B_{y t}\right], \\
& d V_{L t}=V_{L t}\left[\left(r_{t}+\lambda_{L t}\right) d t+d B_{L t}\right],
\end{aligned}
$$

where $\lambda_{x t}, \lambda_{y t}, \lambda_{L t}$ denote the market prices of risk associated with $x, y, L$ shock, respectively. Note that the assumption of a unit volatility is without loss of generality. An artificial market is characterized by the "modifiers" $\nu_{S}, \nu_{H}, \lambda_{L}, \lambda_{x}$, and $\lambda_{y}$.

The modifiers could be quite general stochastic processes, but we focus on the family of artificial markets in which the modifiers are polynomials of time (age), $x$, and $y$. We therefore denote write $\nu_{S t}=\nu_{S}(t, x, y)$ and similar for other quantities, and specify

$$
\begin{aligned}
\nu_{S}(t, x, y)= & \nu_{S, 0, \mathrm{act}}+\nu_{S, 0, \mathrm{ret}}+\nu_{S, 1, \mathrm{act}} t+\nu_{S, 1, \mathrm{ret}} t+\nu_{S, 2} x+\nu_{S, 3} y+\nu_{S, 4} x^{2}+\nu_{S, 5} y^{2}+\nu_{S, 6} x y \\
& +\nu_{S, 7} t^{2}+\nu_{S, 8} x t+\nu_{S, 9} y t \\
\nu_{H}(t, x, y)= & \nu_{H, 0, \mathrm{act}}+\nu_{H, 0, \mathrm{ret}}+\nu_{H, 1, \mathrm{act}} t+\nu_{H, 1, \mathrm{ret}} t+\nu_{H, 2} x+\nu_{H, 3} y+\nu_{H, 4} x^{2}+\nu_{H, 5} y^{2}+\nu_{H, 6} x y \\
& +\nu_{H, 7} t^{2}+\nu_{H, 8} x t+\nu_{H, 9} y t \\
\lambda_{x}(t, x, y)= & \Lambda_{x, 0, \mathrm{act}}+\Lambda_{x, 0, \mathrm{ret}}+\Lambda_{x, 1, \mathrm{act}} t+\Lambda_{x, 1, \mathrm{ret}} t+\Lambda_{x, 2} x+\Lambda_{x, 3} y+\Lambda_{x, 4} x^{2}+\Lambda_{x, 5} y^{2}+\Lambda_{x, 6} x y \\
& +\Lambda_{x, 7} t^{2}+\Lambda_{x, 8} x t+\Lambda_{x, 9} y t \\
\lambda_{y}(t, x, y)= & \Lambda_{y, 0, \mathrm{act}}+\Lambda_{y, 0, \mathrm{ret}}+\Lambda_{y, 1, \mathrm{act}} t+\Lambda_{y, 1, \mathrm{ret}} t+\Lambda_{y, 2} x+\Lambda_{y, 3} y+\Lambda_{y, 4} x^{2}+\Lambda_{y, 5} y^{2}+\Lambda_{y, 6} x y \\
& +\Lambda_{y, 7} t^{2}+\Lambda_{y, 8} x t+\Lambda_{y, 9} y t \\
\lambda_{L}(t, x, y)= & \Lambda_{L, 0, \mathrm{act}}+\Lambda_{L, 0, \mathrm{ret}}+\Lambda_{L, 1, \mathrm{act}} t+\Lambda_{L, 1, \mathrm{ret}} t+\Lambda_{L, 2} x+\Lambda_{L, 3} y+\Lambda_{L, 4} x^{2}+\Lambda_{L, 5} y^{2}+\Lambda_{L, 6} x y \\
& +\Lambda_{L, 7} t^{2}+\Lambda_{L, 8} x t+\Lambda_{L, 9} y t .
\end{aligned}
$$

We refer to such markets as the computable artificial markets since in these markets we can solve the agent's utility maximization in closed form as shown below.

For notational convenience, we define

$$
\mu_{S}^{\prime}(t, x, y)=\mu_{S}(t, x, y)+\bar{D}, \quad \mu_{H}^{\prime}(t, x, y)=\mu_{H}(t, x, y)+R-m
$$

where $\bar{D}$ is the dividend yield, $R$ is the rental rate, and $m$ the maintenance cost rate. By $\Pi_{i t}$ we denote the fraction of wealth invested in asset $i$ at time $t$ with $i \in\{S, H, L, x, y\}$. 


\section{B.2 Human capital in computable artificial markets}

In any of the computable artificial markets, the labor income is spanned and the agent can borrow against future income. By combining these features with the assumed income dynamics, we can compute the human capital - the present value of all future income - by solving a relatively simple partial differential equation (PDE).

Lemma 1 In a computable artificial market, the human capital at time $t$ equals $L_{t} F\left(t, x_{t}, y_{t}\right)$, where $F$ solves the PDE (23) stated in the proof below, with the discrete adjustment $F(\tilde{T}-, x, y)=\Upsilon F(\tilde{T}+, x, y)$ at the retirement date.

Proof: In a complete, unconstrained market we can represent the human capital by the risk-neutral expectation of the future labor income $L_{s}$ discounted by (the integral of) the short-term interest rate $r\left(u, x_{u}, y_{u}\right)$. Let $\mathbb{Q}$ denote the unique risk-neutral probability measure in a given artificial market. To compute the human capital we must therefore identify the $\mathbb{Q}$-dynamics of $L, x$, and $y$. For that purpose we have to identify the market prices of risk associated with the Brownian shocks $B_{S}, B_{H}, B_{L}, B_{x}, B_{y}$. While the market prices of risk associated with $B_{L}, B_{x}, B_{y}$ are $\lambda_{L}(t, x, y), \lambda_{x}(t, x, y), \lambda_{y}(t, x, y)$ by assumption, we identify the market prices of risk $m_{S t}, m_{H t}$ associated with $B_{S}, B_{H}$ by using the fact that the excess expected return on an asset is the product of its sensitivities towards the shocks and the market prices of risks associated with the shocks. For the stock, this means

$$
\mu_{S}^{\prime}(t, x, y)+\chi_{S} x_{t}=\sigma_{S} m_{S t} \Rightarrow m_{S t}=\frac{\mu_{S}^{\prime}(t, x, y)+\chi_{S} x_{t}}{\sigma_{S}}
$$

For an investment in housing units, this implies

$$
\begin{gathered}
\mu_{H}^{\prime}(t, x, y)+\chi_{H x} x_{t}+\chi_{H y} y_{t}=\sigma_{H} \rho_{H S} m_{S t}+\sigma_{H} \hat{\rho}_{H} m_{H t} \Rightarrow \\
m_{H t}=\frac{\mu_{H}^{\prime}(t, x, y)+\chi_{H x} x_{t}+\chi_{H y} y_{t}}{\sigma_{H} \hat{\rho}_{H}}-\frac{\rho_{H S}\left(\mu_{S}^{\prime}(t, x, y)+\chi_{S} x_{t}\right)}{\hat{\rho}_{H} \sigma_{S}} .
\end{gathered}
$$

The risk-neutral income dynamics is therefore

$$
\begin{aligned}
\frac{d L_{t}}{L_{t}}= & {\left[\mu_{L}(t)+\bar{\chi}_{L}(t) y_{t}-\sigma_{L}(t)\left(\rho_{L S} m_{S t}+\hat{\rho}_{L H} m_{H t}+\hat{\rho}_{L} \lambda_{L}(t, x, y)\right)\right] d t } \\
& +\sigma_{L}(t, x, y)\left(\rho_{L S} d B_{S t}^{\mathbb{Q}}+\hat{\rho}_{L H} d B_{H t}^{\mathbb{Q}}+\hat{\rho}_{L} d B_{L t}^{\mathbb{Q}}\right) \\
= & {\left[-\left(M_{L S}(t) \chi_{S}+M_{L H}(t) \chi_{H x}\right) x_{t}-\left(M_{L H}(t) \chi_{H y}-\bar{\chi}_{L}(t)\right) y_{t}+\mu_{L}(t)\right.} \\
& \left.\quad-M_{L S}(t) \mu_{S}^{\prime}(t, x, y)-M_{L H}(t) \mu_{H}^{\prime}(t, x, y)-\sigma_{L}(t) \hat{\rho}_{L} \lambda_{L}(t, x, y)\right] d t \\
+ & \sigma_{L}\left(\rho_{L S} d B_{S t}^{\mathbb{Q}}+\hat{\rho}_{L H} d B_{H t}^{\mathbb{Q}}+\hat{\rho}_{L} d B_{L t}^{\mathbb{Q}}\right),
\end{aligned}
$$


where

$$
M_{L S}(t)=\frac{\sigma_{L}(t)}{\sigma_{S}}\left(\rho_{L S}-\frac{\rho_{H S} \hat{\rho}_{L H}}{\hat{\rho}_{H}}\right), \quad M_{L H}(t)=\frac{\sigma_{L}(t) \hat{\rho}_{L H}}{\sigma_{H} \hat{\rho}_{H}} .
$$

For $x$, we obtain

$$
\begin{aligned}
d x_{t}= & {\left[-\kappa_{x} x_{t}-\sigma_{x}\left(\rho_{x S} m_{S t}+\hat{\rho}_{x H} m_{H t}+\hat{\rho}_{x L} \lambda_{L}(t, x, y)+\hat{\rho}_{x} \lambda_{x}(t, x, y)\right)\right] d t } \\
& +\sigma_{x}\left(\rho_{x S} d B_{S t}^{\mathbb{Q}}+\hat{\rho}_{x H} d B_{H t}^{\mathbb{Q}}+\hat{\rho}_{x L} d B_{L t}^{\mathbb{Q}}+\hat{\rho}_{x} d B_{x t}^{\mathbb{Q}}\right) \\
= & -\left(\kappa_{x}+M_{x S} \chi_{S}+M_{x H} \chi_{H x}\right) x_{t}-M_{x H} \chi_{H y} y_{t} \\
& \left.\quad-M_{x S} \mu_{S}^{\prime}(t, x, y)-M_{x H} \mu_{H}^{\prime}(t, x, y)-\sigma_{x}\left(\hat{\rho}_{x L} \lambda_{L}(t, x, y)+\hat{\rho}_{x} \lambda_{x}(t, x, y)\right)\right] d t \\
+ & \sigma_{x}\left(\rho_{x S} d B_{S t}^{\mathbb{Q}}+\hat{\rho}_{x H} d B_{H t}^{\mathbb{Q}}+\hat{\rho}_{x L} d B_{L t}^{\mathbb{Q}}+\hat{\rho}_{x} d B_{x t}^{\mathbb{Q}}\right),
\end{aligned}
$$

where

$$
M_{x S}=\frac{\sigma_{x}}{\sigma_{S}}\left(\rho_{x S}-\frac{\rho_{H S} \hat{\rho}_{x H}}{\hat{\rho}_{H}}\right), \quad M_{x H}=\frac{\sigma_{x} \hat{\rho}_{x H}}{\sigma_{H} \hat{\rho}_{H}} .
$$

For $y$, we obtain

$$
\begin{aligned}
d y_{t}= & {\left[-\kappa_{y} y_{t}-\sigma_{y}\left(\rho_{y S} m_{S t}+\hat{\rho}_{y H} m_{H t}+\hat{\rho}_{y L} \lambda_{L}(t, x, y)+\hat{\rho}_{y x} \lambda_{x}(t, x, y)+\hat{\rho}_{y} \lambda_{y}(t, x, y)\right)\right] d t } \\
+ & \sigma_{y}\left(\rho_{y S} d B_{S t}^{\mathbb{Q}}+\hat{\rho}_{y H} d B_{H t}^{\mathbb{Q}}+\hat{\rho}_{y L} d B_{L t}^{\mathbb{Q}}+\hat{\rho}_{y x} d B_{x t}^{\mathbb{Q}}+\hat{\rho}_{y} d B_{y t}^{\mathbb{Q}}\right) \\
= & {\left[-\left(M_{y S} \chi_{S}+M_{y H} \chi_{H x}\right) x_{t}-\left(\kappa_{y}+M_{y H} \chi_{H y}\right) y_{t}\right.} \\
& \left.\quad-M_{y S} \mu_{S}^{\prime}(t, x, y)-M_{y H} \mu_{H}^{\prime}(t, x, y)-\sigma_{y}\left(\hat{\rho}_{y L} \lambda_{L}(t, x, y)+\hat{\rho}_{y x} \lambda_{x}(t, x, y)+\hat{\rho}_{y} \lambda_{y}(t, x, y)\right)\right] d t \\
& +\sigma_{y}\left(\rho_{y S} d B_{S t}^{\mathbb{Q}}+\hat{\rho}_{y H} d B_{H t}^{\mathbb{Q}}+\hat{\rho}_{y L} d B_{L t}^{\mathbb{Q}}+\hat{\rho}_{y x} d B_{x t}^{\mathbb{Q}}+\hat{\rho}_{y} d B_{y t}^{\mathbb{Q}}\right),
\end{aligned}
$$

where

$$
M_{y S}=\frac{\sigma_{y}}{\sigma_{S}}\left(\rho_{y S}-\frac{\rho_{H S} \hat{\rho}_{y H}}{\hat{\rho}_{H}}\right), \quad M_{y H}=\frac{\sigma_{y} \hat{\rho}_{y H}}{\sigma_{H} \hat{\rho}_{H}} .
$$

It follows that the human capital in retirement $\mathrm{E}_{t}^{\mathbb{Q}}\left[\int_{t}^{T} e^{-\int_{t}^{s} r\left(u, x_{u}, y_{u}\right) d u} L_{s} d s\right]$ is a function $\mathcal{P}(t, L, x, y)$ and that we can separate it as $\mathcal{P}(t, L, x, y)=L F(t, x, y)$. From general derivatives pricing results we know that if $z=(L, x, y)^{\top}$ and

$$
d z_{t}=\mu_{z}\left(t, z_{t}\right) d t+\Sigma_{z}\left(t, z_{t}\right) d B_{t}^{\mathbb{Q}},
$$


the function $\mathcal{P}(t, z)$ satisfies the partial differential equation (PDE)

$$
\frac{\partial \mathcal{P}}{\partial t}+\frac{\partial \mathcal{P}}{\partial z} \cdot \mu_{z}+\frac{1}{2} \operatorname{tr}\left(\frac{\partial^{2} \mathcal{P}}{\partial z^{2}} \Sigma_{z} \Sigma_{z}^{\top}\right)+L=r \mathcal{P}
$$

and the terminal condition $\mathcal{P}(T, z)=0$. Given the separation $\mathcal{P}=L F$, it follows that $F(t, x, y)$ satisfies the PDE

$$
\begin{aligned}
0=1+ & \frac{\partial F}{\partial t}+\left[-\left(M_{L S}(t) \chi_{S}+M_{L H}(t) \chi_{H x}\right) x+\left(\bar{\chi}_{L}(t)-M_{L H}(t) \chi_{H y}\right) y+\mu_{L}(t)\right. \\
& \left.-M_{L S}(t) \mu_{S}^{\prime}(t, x, y)-M_{L H}(t) \mu_{H}^{\prime}(t, x, y)-\sigma_{L}(t) \hat{\rho}_{L} \lambda_{L}(t, x, y)-r(t, x, y)\right] F \\
+ & {\left[-\left(\kappa_{x}+M_{x S} \chi_{S}+M_{x H} \chi_{H x}\right) x-M_{x H} \chi_{H y} y+\sigma_{x L}(t)\right.} \\
& \left.\quad-M_{x S} \mu_{S}^{\prime}(t, x, y)-M_{x H} \mu_{H}^{\prime}(t, x, y)-\sigma_{x}\left(\hat{\rho}_{x L} \lambda_{L}(t, x, y)+\hat{\rho}_{x} \lambda_{x}(t, x, y)\right)\right] F_{x} \\
+ & {\left[-\left(M_{y S} \chi_{S}+M_{y H} \chi_{H x}\right) x-\left(\kappa_{y}+M_{y H} \chi_{H y}\right) y+\sigma_{y L}(t)\right.} \\
& \left.\quad-M_{y S} \mu_{S}^{\prime}(t, x, y)-M_{y H} \mu_{H}^{\prime}(t, x, y)-\sigma_{y}\left(\hat{\rho}_{y L} \lambda_{L}(t, x, y)+\hat{\rho}_{y x} \lambda_{x}(t, x, y)+\hat{\rho}_{y} \lambda_{y}(t, x, y)\right)\right] F_{y} \\
+ & \frac{1}{2} \sigma_{x}^{2} F_{x x}+\frac{1}{2} \sigma_{y}^{2} F_{y y}+\sigma_{x y} F_{x y},
\end{aligned}
$$

where subscripts on $F$ denote partial derivatives, and where $\sigma_{x L}(t)=\rho_{x L} \sigma_{x} \sigma_{L}(t), \sigma_{y L}(t)=\rho_{y L} \sigma_{y} \sigma_{L}(t)$, and $\sigma_{x y}=\rho_{x y} \sigma_{x} \sigma_{y}$. Given the specification of the interest rate $r(t, x, y)$ in (14), we cannot solve the PDE (23) in closed form, so we solve it backwards from the terminal date $T$ where $F(T, x, y)=0$ using standard finite difference methods.

Before retirement, the human capital is computed from

$$
\mathrm{E}_{t}^{\mathbb{Q}}\left[\int_{t}^{\widetilde{T}} e^{-\int_{t}^{s} r\left(u, x_{u}, y_{u}\right) d u} d s+\int_{\widetilde{T}}^{T} e^{-\int_{t}^{s} r\left(u, x_{u}, y_{u}\right) d u} L_{s} d s\right]
$$

where in the second integral we have to incorporate the drop in income at the retirement time $\widetilde{T}$. We can handle that in the finite difference solution by multiplying the values $F(\tilde{T}+, x, y)$ immediately after retirement by $\Upsilon$ to get the values immediately before retirement.

Given the structure of the PDE (23), it follows that $F$ can be written in the form

$$
F(t, x, y)= \begin{cases}\int_{t}^{T} A(t, s, x, y) d s, & t \in(\widetilde{T}, T], \\ \int_{t}^{\widetilde{T}} A(t, s, x, y) d s+\Upsilon \int_{\widetilde{T}}^{T} A(t, s, x, y) d s, & t \in[0, \widetilde{T}],\end{cases}
$$

and that we can derive some PDE for $A(t, s, x, y)$, but this would still have to be solved numerically and then integrated up numerically. 


\section{B.3 Optimality in computable artificial markets}

The agent's total time $t$ wealth is the sum of tangible wealth and human capital, i.e., $W_{t}+L_{t} F\left(t, x_{t}, y_{t}\right)$. Due to power utility, the indirect utility function is conjectured to have the form $\frac{1}{1-\gamma} G(\cdot)^{\gamma}\left(W_{t}+L_{t} F\left(t, x_{t}, y_{t}\right)\right)^{1-\gamma}$, where $G$ depends on time and on variables driving shifts in investment opportunities (risk-free rate and risk premia) as well as changes in relative prices of consumer goods. In our case, $G$ therefore depends on $x_{t}, y_{t}$, and the house price $H_{t}$. The relative good price $H_{t}$ is expected to enter proportionally with a power (Kraft and Munk 2011) so that $G$ can be separated as $\tilde{k} H^{k} B(t, x, y)$ for appropriate constants $k$ and $\tilde{k}$. These considerations motivate the form of the indirect utility function given below. The optimal strategies then follow from the first-order conditions to the associated Hamilton-Jacobi-Bellman (HJB) equation.

Theorem 1 In a computable artificial market the indirect utility is

$$
J(t, W, H, L, x, y)=\frac{1}{1-\gamma} a^{1-\gamma}\left(\frac{a R H}{1-a}\right)^{(1-a)(1-\gamma)} B(t, x, y)^{\gamma}(W+L F(t, x, y))^{1-\gamma},
$$

where F solves the PDE (23) and B solves the PDE (50) stated in the proof below. The optimal portfolio weights are

$$
\begin{aligned}
\Pi_{S}= & \frac{1}{\gamma \hat{\rho}_{H}^{2} \sigma_{S}^{2}}\left(\mu_{S}^{\prime}(t, x, y)+\chi_{S} x-\frac{\rho_{H S} \sigma_{S}}{\sigma_{H}}\left(\mu_{H}^{\prime}(t, x, y)+\chi_{H x} x+\chi_{H y} y\right)\right) \frac{W+L F}{W} \\
& +\left(M_{x S} \frac{B_{x}}{B}+M_{y S} \frac{B_{y}}{B}\right) \frac{W+L F}{W}-\left(M_{L S}(t)+M_{x S} \frac{F_{x}}{F}+M_{y S} \frac{F_{y}}{F}\right) \frac{L F}{W}, \\
\Pi_{H}= & \frac{1}{\gamma \hat{\rho}_{H}^{2} \sigma_{H}^{2}}\left(\mu_{H}^{\prime}(t, x, y)+\chi_{H x} x+\chi_{H y} y-\frac{\rho_{H S} \sigma_{H}}{\sigma_{S}}\left(\mu_{S}^{\prime}(t, x, y)+\chi_{S} x\right)\right) \frac{W+L F}{W}+k \frac{W+L F}{W} \\
& +\left(M_{x H} \frac{B_{x}}{B}+M_{y H} \frac{B_{y}}{B}\right) \frac{W+L F}{W}-\left(M_{L H}(t)+M_{x H} \frac{F_{x}}{F}+M_{y H} \frac{F_{y}}{F}\right) \frac{L F}{W}, \\
\Pi_{L}= & \frac{1}{\gamma} \lambda_{L}(t, x, y) \frac{W+L F}{W}+\sigma_{L}(t)\left(M_{x L} \frac{B_{x}}{B}+M_{y L} \frac{B_{y}}{B}\right) \frac{W+L F}{W} \\
& -\sigma_{L}(t)\left(\hat{\rho}_{L}+M_{x L} \frac{F_{x}}{F}+M_{y L} \frac{F_{y}}{F}\right) \frac{L F}{W}, \\
\Pi_{x}= & \frac{1}{\gamma} \lambda_{x}(t, x, y) \frac{W+L F}{W}+\sigma_{x}\left(\hat{\rho}_{x} \frac{B_{x}}{B}+M_{y x} \frac{B_{y}}{B}\right) \frac{W+L F}{W}-\sigma_{x}\left(\hat{\rho}_{x} \frac{F_{x}}{F}+M_{y x} \frac{F_{y}}{F}\right) \frac{L F}{W}, \\
\Pi_{y}= & \frac{1}{\gamma} \lambda_{y}(t, x, y) \frac{W+L F}{W}+\sigma_{y} \hat{\rho}_{y} \frac{B_{y}}{B} \frac{W+L F}{W}-\sigma_{y} \hat{\rho}_{y} \frac{F_{y}}{F} \frac{L F}{W},
\end{aligned}
$$

where $k=\frac{(1-a)(\gamma-1)}{\gamma}$ and where the functions and constants $M_{i j}$ were defined in the proof of Lemma 1. The optimal consumption is

$$
\begin{aligned}
c & =a \frac{W+L F}{B}, \\
\phi_{C} & =(1-a) \frac{W+L F}{R H B} .
\end{aligned}
$$

Proof: First we set up the HJB equation, then we conjecture and verify a solution to it. 
Setting up the HJB equation. The wealth dynamics in the artificial market is similar to (7), but adjusted because of the possibility to invest in the artificial asset with price dynamics (15) as well as the modification of $r, \mu_{S}^{\prime}$, and $\mu_{H}^{\prime}$ :

$$
\begin{aligned}
d W_{t}= & \left(W _ { t } \left[r(t, x, y)+\Pi_{S t}\left(\mu_{S}^{\prime}(t, x, y)+\chi_{S} x_{t}\right)+\Pi_{H t}\left(\mu_{H}^{\prime}(t, x, y)+\chi_{H x} x_{t}+\chi_{H y} y_{t}\right)\right.\right. \\
& \left.\left.+\Pi_{L t} \lambda_{L}(t, x, y)+\Pi_{x t} \lambda_{x}(t, x, y)+\Pi_{y t} \lambda_{y}(t, x, y)\right]+\left(L_{t}-c_{t}-\phi_{C t} R H_{t}\right)\right) d t \\
& +W_{t}\left[\left(\Pi_{S t} \sigma_{S}+\Pi_{H t} \sigma_{H} \rho_{H S}\right) d B_{S t}+\Pi_{H t} \sigma_{H} \hat{\rho}_{H} d B_{H t}+\Pi_{L t} d B_{L t}+\Pi_{x t} d B_{x t}+\Pi_{y t} d B_{y t}\right] \\
= & \left(r(t, x, y) W_{t}+\alpha_{t}^{\top} \lambda_{t}+L_{t}-\phi_{C t} R H_{t}-c_{t}\right) d t+\alpha_{t}^{\top} \Sigma d B_{t},
\end{aligned}
$$

where

$$
\begin{gathered}
\alpha_{t}=\left(\begin{array}{c}
\alpha_{S t} \\
\alpha_{H t} \\
\alpha_{L t}
\end{array}\right)=\left(\begin{array}{c}
\Pi_{S t} \sigma_{S} W_{t} \\
\Pi_{H t} \sigma_{H} W_{t} \\
\Pi_{L t} W_{t} \\
\Pi_{x t} W_{t} \\
\Pi_{y t} W_{t}
\end{array}\right), \quad \lambda_{t}=\left(\begin{array}{r}
\left(\mu_{S}^{\prime}(t, x, y)+\chi_{S} x_{t}\right) / \sigma_{S} \\
\left(\mu_{H}^{\prime}(t, x, y)+\chi_{H x} x_{t}+\chi_{H y} y_{t}\right) / \sigma_{H} \\
\lambda_{L}(t, x, y) \\
\lambda_{x}(t, x, y) \\
\lambda_{y}(t, x, y)
\end{array}\right), \\
B_{t}=\left(\begin{array}{c}
B_{S t} \\
B_{H t} \\
B_{L t} \\
B_{x t} \\
B_{y t}
\end{array}\right), \Sigma=\left(\begin{array}{ccccc}
1 & 0 & 0 & 0 & 0 \\
\rho_{H S} & \hat{\rho}_{H} & 0 & 0 & 0 \\
0 & 0 & 1 & 0 & 0 \\
0 & 0 & 0 & 1 & 0 \\
0 & 0 & 0 & 0 & 1
\end{array}\right)
\end{gathered}
$$

Let $Z=(H, L, x, y)^{\top}$ be the vector of state variables, which has the dynamics

$$
d Z_{t}=\mu_{Z}\left(t, Z_{t}\right) d t+\Sigma_{Z}\left(t, Z_{t}\right) d B_{t},
$$

where

$$
\begin{gathered}
\mu_{Z}\left(t, Z_{t}\right)=\left(\begin{array}{c}
H_{t}\left[r(t, x, y)+\mu_{H}(t, x, y)+\chi_{H x} x_{t}+\chi_{H y} y_{t}\right] \\
L_{t}\left[\mu_{L}(t)+\bar{\chi}_{L}(t) y_{t}\right] \\
-\kappa_{x} x_{t} \\
-\kappa_{y} y_{t}
\end{array}\right), \\
\Sigma_{Z}\left(Z_{t}\right)=\left(\begin{array}{ccccc}
H_{t} \sigma_{H} \rho_{H S} & H_{t} \sigma_{H} \hat{\rho}_{H} & 0 & 0 & 0 \\
L_{t} \sigma_{L}(t) \rho_{L S} & L_{t} \sigma_{L}(t) \hat{\rho}_{L H} & L_{t} \sigma_{L}(t) \hat{\rho}_{L} & 0 & 0 \\
\sigma_{x} \rho_{x S} & \sigma_{x} \hat{\rho}_{x H} & \sigma_{x} \hat{\rho}_{x L} & \sigma_{x} \hat{\rho}_{x} & 0 \\
\sigma_{y} \rho_{y S} & \sigma_{y} \hat{\rho}_{y H} & \sigma_{y} \hat{\rho}_{y L} & \sigma_{y} \hat{\rho}_{y x} & \sigma_{y} \hat{\rho}_{y}
\end{array}\right) .
\end{gathered}
$$


The Hamilton-Jacobi-Bellman equation (HJB) associated with the problem can be written as

$$
\delta J=\mathcal{L}_{1} J+\mathcal{L}_{2} J+\mathcal{L}_{3} J
$$

where

$$
\begin{aligned}
& \mathcal{L}_{1} J=\max _{c, \phi_{C}}\left\{U\left(c, \phi_{C}\right)-J_{W}\left(c+H R \phi_{C}\right)\right\}, \\
& \mathcal{L}_{2} J=\max _{\alpha}\left\{J_{W} \alpha^{\top} \lambda+\frac{1}{2} J_{W W} \alpha^{\top} \Sigma \Sigma^{\top} \alpha+\alpha^{\top} \Sigma \Sigma_{Z}^{\top} J_{W Z}\right\}, \\
& \mathcal{L}_{3} J=\frac{\partial J}{\partial t}+J_{W}(r W+L)+J_{Z}^{\top} \mu_{Z}+\frac{1}{2} \operatorname{trace}\left(J_{Z Z} \Sigma_{Z} \Sigma_{Z}^{\top}\right) .
\end{aligned}
$$

Recall that $J=J(t, W, H, L, x, y)=J(t, W, Z)$ so that

$$
J_{Z}=\left(\begin{array}{c}
J_{H} \\
J_{L} \\
J_{x} \\
J_{y}
\end{array}\right), \quad J_{Z Z}=\left(\begin{array}{cccc}
J_{H H} & J_{H L} & J_{H x} & J_{H y} \\
J_{H L} & J_{L L} & J_{L x} & J_{L y} \\
J_{H x} & J_{L x} & J_{x x} & J_{x y} \\
J_{H y} & J_{L y} & J_{x y} & J_{y y}
\end{array}\right), \quad J_{W Z}=\left(\begin{array}{c}
J_{W H} \\
J_{W L} \\
J_{W x} \\
J_{W y}
\end{array}\right)
$$

First, consider $\mathcal{L}_{1} J$. The first-order conditions are $U_{c}\left(c^{*}, \phi_{C}^{*}\right)=J_{W}$ and $U_{\phi}\left(c^{*}, \phi_{C}^{*}\right)=R H J_{W}$. These imply $U_{\phi}\left(c^{*}, \phi_{C}^{*}\right) / U_{c}\left(c^{*}, \phi_{C}^{*}\right)=R H$ so that

$$
\phi_{C}^{*}=c^{*}\left(\frac{a R H}{1-a}\right)^{-1} .
$$

We substitute that relation into $U_{c}=J_{W}$ and find

$$
c^{*}=J_{W}^{-1 / \gamma} a^{1 / \gamma}\left(\frac{a R H}{1-a}\right)^{k},
$$

and hence

$$
\phi_{C}^{*}=J_{W}^{-1 / \gamma} a^{1 / \gamma}\left(\frac{a R H}{1-a}\right)^{k-1}
$$

where $k=(1-a)(\gamma-1) / \gamma$. These maximizers lead to

$$
\mathcal{L}_{1} J=\frac{\gamma}{1-\gamma} J_{W}^{\frac{\gamma-1}{\gamma}} a^{\frac{1-\gamma}{\gamma}}\left(\frac{a R H}{1-a}\right)^{k}
$$

Next, consider $\mathcal{L}_{2} J$. The first-order condition for $\alpha$ reads $J_{W} \lambda+J_{W W} \Sigma \Sigma^{\top} \alpha+\Sigma \Sigma_{Z}^{\top} J_{W Z}=0$ or

$$
\alpha=-\frac{J_{W}}{J_{W W}}\left(\Sigma \Sigma^{\top}\right)^{-1} \lambda-\frac{1}{J_{W W}}\left(\Sigma \Sigma^{\top}\right)^{-1} \Sigma \Sigma_{Z}^{\top} J_{W Z}=-\frac{J_{W}}{J_{W W}}\left(\Sigma \Sigma^{\top}\right)^{-1} \lambda-\frac{1}{J_{W W}}\left(\Sigma_{Z} \Sigma^{-1}\right)^{\top} J_{W Z} .
$$


Substituting the optimal $\alpha$ back into $\mathcal{L}_{2} J$ leads to

$$
\mathcal{L}_{2} J=-\frac{1}{2} \frac{J_{W}^{2}}{J_{W W}} \lambda^{\top}\left(\Sigma \Sigma^{\top}\right)^{-1} \lambda-\frac{J_{W}}{J_{W W}} J_{W Z}^{\top} \Sigma_{Z} \Sigma^{-1} \lambda-\frac{1}{2} \frac{1}{J_{W W}} J_{W Z}^{\top} \Sigma_{Z} \Sigma_{Z}^{\top} J_{W Z}
$$

The matrix products are

$$
\begin{aligned}
& \Sigma \Sigma^{\top}=\left(\begin{array}{ccccc}
1 & \rho_{H S} & 0 & 0 & 0 \\
\rho_{H S} & 1 & 0 & 0 & 0 \\
0 & 0 & 1 & 0 & 0 \\
0 & 0 & 0 & 1 & 0 \\
0 & 0 & 0 & 0 & 1
\end{array}\right) \Rightarrow\left(\Sigma \Sigma^{\top}\right)^{-1}=\frac{1}{\hat{\rho}_{H}^{2}}\left(\begin{array}{ccccc}
1 & -\rho_{H S} & 0 & 0 & 0 \\
-\rho_{H S} & 1 & 0 & 0 & 0 \\
0 & 0 & \hat{\rho}_{H}^{2} & 0 & 0 \\
0 & 0 & 0 & \hat{\rho}_{H}^{2} & 0 \\
0 & 0 & 0 & 0 & \hat{\rho}_{H}^{2}
\end{array}\right), \\
& \Sigma_{Z} \Sigma^{-1}=\left(\begin{array}{ccccc}
0 & H \sigma_{H} & 0 & 0 & 0 \\
L M_{L S} \sigma_{S} & L M_{L H} \sigma_{H} & L \hat{\rho}_{L} \sigma_{L} & 0 & 0 \\
M_{x S} \sigma_{S} & M_{x H} \sigma_{H} & \hat{\rho}_{x L} \sigma_{x} & \hat{\rho}_{x} \sigma_{x} & 0 \\
M_{y S} \sigma_{S} & M_{y H} \sigma_{H} & \hat{\rho}_{y L} \sigma_{y} & \hat{\rho}_{y x} \sigma_{y} & \hat{\rho}_{y} \sigma_{y}
\end{array}\right), \quad \Sigma_{Z} \Sigma_{Z}^{\top}=\left(\begin{array}{cccc}
H^{2} \sigma_{H}^{2} & H L \sigma_{H L} & H \sigma_{H x} & H \sigma_{H y} \\
H L \sigma_{H L} & L^{2} \sigma_{L}^{2} & L \sigma_{L x} & L \sigma_{L y} \\
H \sigma_{H x} & L \sigma_{L x} & \sigma_{x}^{2} & \sigma_{x y} \\
H \sigma_{H y} & L \sigma_{L y} & \sigma_{x y} & \sigma_{y}^{2}
\end{array}\right)
\end{aligned}
$$

where we have applied the covariance notation $\sigma_{a b}=\rho_{a b} \sigma_{a} \sigma_{b}$ and constants defined in Appendix B.2.

Substitution of these matrix products into $(36)$ gives

$$
\begin{aligned}
\alpha_{S}= & -\frac{J_{W}}{J_{W W}} \frac{1}{\sigma_{S} \hat{\rho}_{H}^{2}}\left(\mu_{S}^{\prime}(t, x, y)+\chi_{S} x-\frac{\rho_{H S} \sigma_{S}}{\sigma_{H}}\left[\mu_{H}^{\prime}(t, x, y)+\chi_{H x} x+\chi_{H y} y\right]\right) \\
& -\frac{J_{W L}}{J_{W W}} L M_{L S}(t) \sigma_{S}-\frac{J_{W x}}{J_{W W}} M_{x S} \sigma_{S}-\frac{J_{W y}}{J_{W W}} M_{y S} \sigma_{S}, \\
\alpha_{H}= & -\frac{J_{W}}{J_{W W}} \frac{1}{\sigma_{H} \hat{\rho}_{H}^{2}}\left(\mu_{H}^{\prime}(t, x, y)+\chi_{H x} x+\chi_{H y} y-\frac{\rho_{H S} \sigma_{H}}{\sigma_{S}}\left[\mu_{S}^{\prime}(t, x, y)+\chi_{S} x\right]\right) \\
& -\frac{J_{W H}}{J_{W W}} H \sigma_{H}-\frac{J_{W L}}{J_{W W}} L M_{L H}(t) \sigma_{H}-\frac{J_{W x}}{J_{W W}} M_{x H} \sigma_{H}-\frac{J_{W y}}{J_{W W}} M_{y H} \sigma_{H}, \\
\alpha_{L}= & -\frac{J_{W}}{J_{W W}} \lambda_{L}(t, x, y)-\frac{J_{W L}}{J_{W W}} L \hat{\rho}_{L} \sigma_{L}(t)-\frac{J_{W x}}{J_{W W}} \hat{\rho}_{x L} \sigma_{x}-\frac{J_{W y}}{J_{W W}} \hat{\rho}_{y L} \sigma_{y}, \\
\alpha_{x}= & -\frac{J_{W}}{J_{W W}} \lambda_{x}(t, x, y)-\frac{J_{W x}}{J_{W W}} \hat{\rho}_{x} \sigma_{x}-\frac{J_{W y}}{J_{W W}} \hat{\rho}_{y x} \sigma_{y}, \\
\alpha_{y}= & -\frac{J_{W}}{J_{W W}} \lambda_{y}(t, x, y)-\frac{J_{W y}}{J_{W W}} \hat{\rho}_{y} \sigma_{y} .
\end{aligned}
$$

Conjecture of the solution to the HJB equation. We conjecture

$$
J(t, W, H, L, x, y)=\frac{1}{1-\gamma} G(t, H, x, y)^{\gamma}(W+L F(t, x, y))^{1-\gamma}
$$

It turns out to be useful to express the derivatives of $J$ in terms of $J$ itself:

$$
J_{W}=\frac{(1-\gamma) J}{W+L F}, \quad J_{W W}=-\frac{\gamma(1-\gamma) J}{(W+L F)^{2}}
$$




$$
\begin{aligned}
& J_{L}=(1-\gamma) J \frac{F}{W+L F}, \\
& J_{L L}=-\gamma(1-\gamma) J \frac{F^{2}}{(W+L F)^{2}}, \\
& J_{H}=\gamma J \frac{G_{H}}{G}, \\
& J_{W L}=-\gamma(1-\gamma) J \frac{F}{(W+L F)^{2}}, \\
& J_{H H}=\gamma(1-\gamma) J\left[\frac{1}{1-\gamma} \frac{G_{H H}}{G}-\left(\frac{G_{H}}{G}\right)^{2}\right] \text {, } \\
& J_{H L}=\gamma(1-\gamma) J \frac{G_{H}}{G} \frac{F}{W+L F}, \\
& J_{W H}=\gamma(1-\gamma) J \frac{1}{W+L F} \frac{G_{H}}{G}, \\
& J_{y}=(1-\gamma) J\left[\frac{\gamma}{1-\gamma} \frac{G_{y}}{G}+\frac{L F_{y}}{W+L F}\right], \\
& J_{x}=(1-\gamma) J\left[\frac{\gamma}{1-\gamma} \frac{G_{x}}{G}+\frac{L F_{x}}{W+L F}\right] \text {, } \\
& J_{W y}=\gamma(1-\gamma) J\left[\frac{G_{y}}{G} \frac{1}{W+L F}-\frac{L F_{y}}{(W+L F)^{2}}\right] \text {, } \\
& J_{W x}=\gamma(1-\gamma) J\left[\frac{G_{x}}{G} \frac{1}{W+L F}-\frac{L F_{x}}{(W+L F)^{2}}\right] \text {, } \\
& J_{x x}=(1-\gamma) J\left[\frac{\gamma}{1-\gamma} \frac{G_{x x}}{G}-\gamma\left(\frac{G_{x}}{G}\right)^{2}+2 \gamma \frac{G_{x}}{G} \frac{L F_{x}}{W+L F}-\gamma\left(\frac{L F_{x}}{W+L F}\right)^{2}+\frac{L F_{x x}}{W+L F}\right] \text {, } \\
& J_{y y}=(1-\gamma) J\left[\frac{\gamma}{1-\gamma} \frac{G_{y y}}{G}-\gamma\left(\frac{G_{y}}{G}\right)^{2}+2 \gamma \frac{G_{y}}{G} \frac{L F_{y}}{W+L F}-\gamma\left(\frac{L F_{y}}{W+L F}\right)^{2}+\frac{L F_{y y}}{W+L F}\right] \text {, } \\
& J_{H x}=\gamma(1-\gamma) J\left[\frac{1}{1-\gamma} \frac{G_{H x}}{G}-\frac{G_{x} G_{H}}{G^{2}}+\frac{L F_{x}}{W+L F} \frac{G_{H}}{G}\right] \text {, } \\
& J_{H y}=\gamma(1-\gamma) J\left[\frac{1}{1-\gamma} \frac{G_{H y}}{G}-\frac{G_{y} G_{H}}{G^{2}}+\frac{L F_{y}}{W+L F} \frac{G_{H}}{G}\right] \text {, } \\
& J_{L x}=(1-\gamma) J\left[\gamma \frac{G_{x}}{G} \frac{F}{W+L F}+\frac{F_{x}}{W+L F}-\gamma \frac{L F F_{x}}{(W+L F)^{2}}\right], \\
& J_{L y}=(1-\gamma) J\left[\gamma \frac{G_{y}}{G} \frac{F}{W+L F}+\frac{F_{y}}{W+L F}-\gamma \frac{L F F_{y}}{(W+L F)^{2}}\right] \text {, } \\
& J_{x y}=(1-\gamma) J\left[\frac{\gamma}{1-\gamma} \frac{G_{x y}}{G}-\gamma \frac{G_{x} G_{y}}{G^{2}}+\gamma \frac{G_{x}}{G} \frac{L F_{y}}{W+L F}+\gamma \frac{G_{y}}{G} \frac{L F_{x}}{W+L F}+\frac{L F_{x y}}{W+L F}-\gamma \frac{L^{2} F_{x} F_{y}}{(W+L F)^{2}}\right] .
\end{aligned}
$$

Next, we rewrite the terms $\mathcal{L}_{1} J, \mathcal{L}_{2} J, \mathcal{L}_{3} J$ exploiting the conjecture for $J$. First, since

$$
J_{w}^{\frac{\gamma-1}{\gamma}}=J_{w} J_{w}^{-1 / \gamma}=\frac{(1-\gamma) J}{W+L F}\left\{G^{\gamma}[W+L F]^{-\gamma}\right\}^{-1 / \gamma}=(1-\gamma) J \frac{1}{G}
$$

we get from (35) that

$$
\mathcal{L}_{1} J=\gamma J \frac{1}{G} a^{\frac{1-\gamma}{\gamma}}\left(\frac{a R H}{1-a}\right)^{k}
$$

Next, we have from (37) that

$$
\mathcal{L}_{2} J=\mathcal{L}_{2,1} J+\mathcal{L}_{2,2} J+\mathcal{L}_{2,3} J
$$


where

$$
\begin{aligned}
\mathcal{L}_{2,1} J= & -\frac{1}{2} \frac{J_{W}^{2}}{J_{W W}} \lambda^{\top}\left(\Sigma \Sigma^{\top}\right)^{-1} \lambda=\frac{1-\gamma}{2 \gamma} J \lambda^{\top}\left(\Sigma \Sigma^{\top}\right)^{-1} \lambda \\
= & \frac{1-\gamma}{2 \gamma} J\left\{\left(\frac{\mu_{S}^{\prime}(t, x, y)+\chi_{S} x_{t}}{\sigma_{S} \hat{\rho}_{H}}\right)^{2}+\left(\frac{\mu_{H}^{\prime}(t, x, y)+\chi_{H x} x_{t}+\chi_{H y} y_{t}}{\sigma_{H} \hat{\rho}_{H}}\right)^{2}\right. \\
& \left.\quad-2 \rho_{H S} \frac{\mu_{S}^{\prime}(t, x, y)+\chi_{S} x_{t}}{\sigma_{S} \hat{\rho}_{H}} \frac{\mu_{H}^{\prime}(t, x, y)+\chi_{H x} x_{t}+\chi_{H y} y_{t}}{\sigma_{H} \hat{\rho}_{H}}+\lambda_{L}(t, x, y)^{2}+\lambda_{x}(t, x, y)^{2}+\lambda_{y}(t, x, y)^{2}\right\}
\end{aligned}
$$

and

$$
\begin{aligned}
& \mathcal{L}_{2,2} J=-\frac{J_{W}}{J_{W W}} J_{W Z}^{\top} \Sigma_{Z} \Sigma^{-1} \lambda=\frac{1}{\gamma}(W+L F) J_{W Z}^{\top} \Sigma_{Z} \Sigma^{-1} \lambda \\
& =(1-\gamma) J\left(\begin{array}{c}
\frac{G_{H}}{G} \\
-\frac{F}{W+L F} \\
\frac{G_{x}}{G}-\frac{L F_{x}}{W+L F} \\
\frac{G_{y}}{G}-\frac{L F_{y}}{W+L F}
\end{array}\right)^{\top}\left(\begin{array}{ccccc}
0 & H \sigma_{H} & 0 & 0 & 0 \\
L \ell(t) \sigma_{S} & L M_{L H}(t) \sigma_{H} & L \hat{\rho}_{L} \sigma_{L}(t) & 0 & 0 \\
M_{x S} \sigma_{S} & M_{x H} \sigma_{H} & \hat{\rho}_{x L} \sigma_{x} & \hat{\rho}_{x} \sigma_{x} & 0 \\
M_{y S} \sigma_{S} & M_{y H} \sigma_{H} & \hat{\rho}_{y L} \sigma_{y} & \hat{\rho}_{y x} \sigma_{y} & \hat{\rho}_{y} \sigma_{y}
\end{array}\right)\left(\begin{array}{c}
\frac{\mu_{S}^{\prime}(t, x, y)+\chi_{S} x_{t}}{\sigma_{S}} \\
\frac{\mu_{H}^{\prime}(t, x, y)+\chi_{H x} x_{t}+\chi_{H y} y_{t}}{\sigma_{H}} \\
\lambda_{L}(t, x, y) \\
\lambda_{x}(t, x, y) \\
\lambda_{y}(t, x, y)
\end{array}\right) \\
& =(1-\gamma) J\left\{\frac{H G_{H}}{G}\left(\mu_{H}^{\prime}(t, x, y)+\chi_{H x} x_{t}+\chi_{H y} y_{t}\right)+\frac{G_{x}}{G}\left(-\left(M_{x S} \chi_{S}+M_{x H} \chi_{H x}\right) x_{t}-M_{x H} \chi_{H y} y_{t}\right.\right. \\
& \left.-M_{x S} \mu_{S}^{\prime}(t, x, y)-M_{x H} \mu_{H}^{\prime}(t, x, y)-\sigma_{x}\left(\hat{\rho}_{x L} \lambda_{L}(t, x, y)+\hat{\rho}_{x} \lambda_{x}(t, x, y)\right)\right) \\
& +\frac{G_{y}}{G}\left(-\left(M_{y S} \chi_{S}+M_{y H} \chi_{H x}\right) x_{t}-M_{y H} \chi_{H y} y_{t}\right. \\
& \left.-M_{y S} \mu_{S}^{\prime}(t, x, y)-M_{y H} \mu_{H}^{\prime}(t, x, y)-\sigma_{y}\left(\hat{\rho}_{y L} \lambda_{L}(t, x, y)+\hat{\rho}_{y x} \lambda_{x}(t, x, y)+\hat{\rho}_{y} \lambda_{y}(t, x, y)\right)\right) \\
& -\frac{L}{W+L F}\left[F \left(-\left(M_{L S}(t) \chi_{S}+M_{L H}(t) \chi_{H x}\right) x_{t}+\left(\operatorname{bar} \chi_{y L}(t)-M_{L H}(t) \chi_{H y}\right) y_{t}+\mu_{L}(t)-r(t)\right.\right. \\
& \left.-M_{L S}(t) \mu_{S}^{\prime}(t, x, y)+M_{L H}(t) \mu_{H}^{\prime}(t, x, y)-\sigma_{L}(t) \hat{\rho}_{L} \lambda_{L}(t, x, y)\right) \\
& +F_{x}\left(-\left(M_{x S} \chi_{S}+M_{x H} \chi_{H x}\right) x_{t}-M_{x H} \chi_{H y} y_{t}\right. \\
& \left.-M_{x S} \mu_{S}^{\prime}(t, x, y)-M_{x H} \mu_{H}^{\prime}(t, x, y)-\sigma_{x}\left(\hat{\rho}_{x L} \lambda_{L}(t, x, y)+\hat{\rho}_{x} \lambda_{x}(t, x, y)\right)\right) \\
& +F_{y}\left(-\left(M_{y S} \chi_{S}+M_{y H} \chi_{H x}\right) x_{t}-M_{y H} \chi_{H y} y_{t}-M_{y S} \mu_{S}^{\prime}(t, x, y)-M_{y H} \mu_{H}^{\prime}(t, x, y)\right. \\
& \left.\left.\left.-\sigma_{y}\left(\hat{\rho}_{y L} \lambda_{L}(t, x, y)+\hat{\rho}_{y x} \lambda_{x}(t, x, y)+\hat{\rho}_{y} \lambda_{y}(t, x, y)\right)\right)\right]\right\}
\end{aligned}
$$

and, furthermore,

$$
\mathcal{L}_{2,3} J=-\frac{1}{2} \frac{1}{J_{W W}} J_{W Z}^{\top} \Sigma_{Z} \Sigma_{Z}^{\top} J_{W Z}
$$




$$
\begin{aligned}
& =\frac{1}{2} \gamma(1-\gamma) J\left(\begin{array}{c}
\frac{G_{H}}{G} \\
-\frac{F}{W+L F} \\
\frac{G_{x}}{G}-\frac{L F_{x}}{W+L F} \\
\frac{G_{y}}{G}-\frac{L F_{y}}{W+L F}
\end{array}\right)\left(\begin{array}{cccc}
H^{2} \sigma_{H}^{2} & H L \sigma_{H L} & H \sigma_{H x} & H \sigma_{H y} \\
H L \sigma_{H L} & L^{2} \sigma_{L}^{2} & L \sigma_{L x} & L \sigma_{L y} \\
H \sigma_{H x} & L \sigma_{L x} & \sigma_{x}^{2} & \sigma_{x y} \\
H \sigma_{H y} & L \sigma_{L y} & \sigma_{x y} & \sigma_{y}^{2}
\end{array}\right)\left(\begin{array}{c}
\frac{G_{H}}{G} \\
-\frac{F}{W+L F} \\
\frac{G_{x}}{G}-\frac{L F_{x}}{W+L F} \\
\frac{G_{y}}{G}-\frac{L F_{y}}{W+L F}
\end{array}\right) \\
& =\gamma(1-\gamma) J\left\{\frac{1}{2} \sigma_{H}^{2} \frac{H^{2} G_{H}^{2}}{G^{2}}+\frac{1}{2} \sigma_{x}^{2} \frac{G_{x}^{2}}{G^{2}}+\frac{1}{2} \sigma_{y}^{2} \frac{G_{y}^{2}}{G^{2}}+\sigma_{H x} \frac{H G_{H} G_{x}}{G^{2}}+\sigma_{H y} \frac{H G_{H} G_{y}}{G^{2}}+\sigma_{x y} \frac{G_{x} G_{y}}{G^{2}}\right. \\
& -\frac{L}{W+L F}\left[\frac{H G_{H}}{G}\left(\sigma_{H L} F+\sigma_{H x} F_{x}+\sigma_{H y} F_{y}\right)\right. \\
& \left.+\frac{G_{x}}{G}\left(\sigma_{L x} F+\sigma_{x}^{2} F_{x}+\sigma_{x y} F_{y}\right)+\frac{G_{y}}{G}\left(\sigma_{L y} F+\sigma_{x y} F_{x}+\sigma_{y}^{2} F_{y}\right)\right] \\
& \left.+\frac{L^{2}}{(W+L F)^{2}}\left[\frac{1}{2} \sigma_{L}^{2} F^{2}+\frac{1}{2} \sigma_{x}^{2} F_{x}^{2}+\frac{1}{2} \sigma_{y}^{2} F_{y}^{2}+\sigma_{L x} F F_{x}+\sigma_{L y} F F_{y}+\sigma_{x y} F_{x} F_{y}\right]\right\} .
\end{aligned}
$$

Finally, we can rewrite $\mathcal{L}_{3} J$ as

$$
\mathcal{L}_{3} J=\mathcal{L}_{3,1} J+\mathcal{L}_{3,2} J,
$$

where

$$
\begin{aligned}
\mathcal{L}_{3,1} J=\frac{\partial J}{\partial t}+J_{W}(r(t, x, y) W+L)+J_{Z}^{\top} \mu_{Z} \\
=(1-\gamma) J\left\{r(t, x, y)+\frac{\gamma}{1-\gamma}\left[\frac{1}{G} \frac{\partial G}{\partial t}+\frac{H G_{H}}{G}\left(r(t, x, y)+\mu_{H}(t, x, y)+\chi_{H x} x_{t}+\chi_{H y} y_{t}\right)-\kappa_{x} x_{t} \frac{G_{x}}{G}-\kappa_{y} y_{t} \frac{G_{y}}{G}\right]\right. \\
\left.\quad+\frac{L}{W+L F}\left[\frac{\partial F}{\partial t}+1+\left(\mu_{L}(t)+\bar{\chi}_{L}(t) y_{t}-r(t, x, y)\right) F-\kappa_{x} x_{t} F_{x}-\kappa_{y} y_{t} F_{y}\right]\right\}
\end{aligned}
$$

and

$$
\begin{aligned}
\mathcal{L}_{3,2} J= & \frac{1}{2} \operatorname{trace}\left(J_{Z Z} \Sigma_{Z} \Sigma_{Z}^{\top}\right) \\
= & \frac{1}{2} \sigma_{H}^{2} H^{2} J_{H H}+\frac{1}{2} \sigma_{L}^{2} L^{2} J_{L L}+\frac{1}{2} \sigma_{x}^{2} J_{x x}+\frac{1}{2} \sigma_{y}^{2} J_{y y}+\sigma_{H L} H L J_{H L}+\sigma_{H x} H J_{H x} \\
& +\sigma_{H y} H J_{H y}+\sigma_{L x} L J_{L x}+\sigma_{L y} L J_{L y}+\sigma_{x y} J_{x y}+\sigma_{x z} J_{x z}+\sigma_{y z} J_{y z} \\
= & (1-\gamma) J\left\{\frac { \gamma } { 1 - \gamma } \left[\frac{1}{2} \sigma_{H}^{2} H^{2} \frac{G_{H H}}{G}+\frac{1}{2} \sigma_{x}^{2} \frac{G_{x x}}{G}+\frac{1}{2} \sigma_{y}^{2} \frac{G_{y y}}{G}-\frac{1-\gamma}{2} \sigma_{H}^{2} H^{2} \frac{G_{H}^{2}}{G^{2}}-\frac{1-\gamma}{2} \sigma_{x}^{2} \frac{G_{x}^{2}}{G^{2}}\right.\right. \\
& \quad-\frac{1-\gamma}{2} \sigma_{y}^{2} \frac{G_{y}^{2}}{G^{2}}+\sigma_{H x} H \frac{G_{H x}}{G}+\sigma_{H y} H \frac{G_{H y}}{G}+\sigma_{x y} \frac{G_{x y}}{G} \\
& \left.\quad-(1-\gamma) \sigma_{H x} H \frac{G_{H} G_{x}}{G^{2}}-(1-\gamma) \sigma_{H y} H \frac{G_{H} G_{y}}{G^{2}}-(1-\gamma) \sigma_{x y} \frac{G_{x} G_{y}}{G^{2}}\right] \\
\left.+\frac{L}{W+}\right] & {\left[\frac{1}{2} \sigma_{x}^{2} F_{x x}+\frac{1}{2} \sigma_{y}^{2} F_{y y}+\sigma_{x y} F_{x y}+\sigma_{L x} F_{x}+\sigma_{L y} F_{y}\right.}
\end{aligned}
$$




$$
\begin{aligned}
&+\gamma[ \frac{H G_{H}}{G}\left(\sigma_{H L} F+\sigma_{H x} F_{x}+\sigma_{H y} F_{y}\right) \\
&\left.\left.+\frac{G_{x}}{G}\left(\sigma_{L x} F+\sigma_{x}^{2} F_{x}+\sigma_{x y} F_{y}\right)+\frac{G_{y}}{G}\left(\sigma_{L y} F+\sigma_{x y} F_{x}+\sigma_{y}^{2} F_{y}\right)\right]\right] \\
&\left.-\frac{L^{2}}{(W+L F)^{2}} \gamma\left[\frac{1}{2} \sigma_{L}^{2} F^{2}+\frac{1}{2} \sigma_{x}^{2} F_{x}^{2}+\frac{1}{2} \sigma_{y}^{2} F_{y}^{2}+\sigma_{L x} F F_{x}+\sigma_{L y} F F_{y}+\sigma_{x y} F_{x} F_{y}\right]\right\}
\end{aligned}
$$

By adding $\mathcal{L}_{2,3} J$ and $\mathcal{L}_{3,2} J$ numerous terms cancel so that we are left with

$$
\begin{aligned}
\mathcal{L}_{2,3} J+\mathcal{L}_{3,2} J=(1-\gamma) J\{ & \frac{\gamma}{1-\gamma}\left[\frac{1}{2} \sigma_{H}^{2} H^{2} \frac{G_{H H}}{G}+\frac{1}{2} \sigma_{x}^{2} \frac{G_{x x}}{G}+\frac{1}{2} \sigma_{y}^{2} \frac{G_{y y}}{G}+\sigma_{H x} H \frac{G_{H x}}{G}+\sigma_{H y} H \frac{G_{H y}}{G}+\sigma_{x y} \frac{G_{x y}}{G}\right] \\
& \left.+\frac{L}{W+L F}\left[\frac{1}{2} \sigma_{x}^{2} F_{x x}+\frac{1}{2} \sigma_{y}^{2} F_{y y}+\sigma_{x y} F_{x y}+\sigma_{L x} F_{x}+\sigma_{L y} F_{y}\right]\right\} .
\end{aligned}
$$

If we further add $\mathcal{L}_{2,2} J$ and $\mathcal{L}_{3,1} J$ to this, all the terms multiplying $L /(W+L F)$ cancel because $F$ satisfies the PDE (23). In sum, we get

$$
\begin{aligned}
& \delta J= \mathcal{L}_{1} J+\mathcal{L}_{2,1} J+\mathcal{L}_{2,2} J+\mathcal{L}_{2,3} J+\mathcal{L}_{3,1} J+\mathcal{L}_{3,2} J \\
&=\gamma J \frac{1}{G} a^{\frac{1-\gamma}{\gamma}}\left(\frac{a R H}{1-a}\right)^{k} \\
&+J \frac{1-\gamma}{2 \gamma}\left[\left(\frac{\mu_{S}^{\prime}(t, x, y)+\chi_{S} x_{t}}{\sigma_{S} \hat{\rho}_{H}}\right)^{2}+\left(\frac{\mu_{H}^{\prime}(t, x, y)+\chi_{H x} x_{t}+\chi_{H y} y_{t}}{\sigma_{H} \hat{\rho}_{H}}\right)^{2}\right. \\
&\left.\quad-2 \rho_{H S} \frac{\mu_{S}^{\prime}(t, x, y)+\chi_{S} x_{t}}{\sigma_{S} \hat{\rho}_{H}} \frac{\mu_{H}^{\prime}(t, x, y)+\chi_{H x} x_{t}+\chi_{H y} y_{t}}{\sigma_{H} \hat{\rho}_{H}}+\lambda_{L}(t, x, y)^{2}+\lambda_{x}(t, x, y)^{2}+\lambda_{y}(t, x, y)^{2}\right] \\
&+(1-\gamma) J \frac{1}{G}\left\{r(t, x, y)+H G_{H}\left(\mu_{H}^{\prime}(t, x, y)+\chi_{H x} x_{t}+\chi_{H y} y_{t}\right)+\frac{G_{x}}{G}\left(-\left(M_{x S} \chi_{S}+M_{x H} \chi_{H x}\right) x_{t}-M_{x H} \chi_{H y} y_{t}\right.\right. \\
&\left.\quad-M_{x S} \mu_{S}^{\prime}(t, x, y)-M_{x H} \mu_{H}^{\prime}(t, x, y)-\sigma_{x}\left(\hat{\rho}_{x L} \lambda_{L}(t, x, y)+\hat{\rho}_{x} \lambda_{x}(t, x, y)\right)\right) \\
& \quad \frac{G_{y}}{G}\left(-\left(M_{y S} \chi_{S}+M_{y H} \chi_{H x}\right) x_{t}-M_{y H} \chi_{H y} y_{t}\right. \\
&\left.\quad-M_{y S} \mu_{S}^{\prime}(t, x, y)-M_{y H} \mu_{H}^{\prime}(t, x, y)-\sigma_{y}\left(\hat{\rho}_{y L} \lambda_{L}(t, x, y)+\hat{\rho}_{y x} \lambda_{x}(t, x, y)+\hat{\rho}_{y} \lambda_{y}(t, x, y)\right)\right) \\
& \quad \frac{1}{1-\gamma}\left[\frac{1}{2} \sigma_{H}^{2} H^{2} G_{H H}+\frac{1}{2} \sigma_{x}^{2} G_{x x}+\frac{1}{2} \sigma_{y}^{2} G_{y y}+\sigma_{H x} H G_{H x}+\sigma_{H y} H G_{H y}+\sigma_{x y} G_{x y}\right. \\
&\left.\left.\quad+\frac{\partial G}{\partial t}+H G_{H}\left(r(t, x, y)+\mu_{H}(t, x, y)+\chi_{H x} x_{t}+\chi_{H y} y_{t}\right)-\kappa_{x} x_{t} G_{x}-\kappa_{y} y_{t} G_{y}\right]\right\}
\end{aligned}
$$

Therefore, it follows that the HJB equation is satisfied if the $G$ function solves the PDE

$$
\begin{aligned}
0= & \tilde{k} H^{k}+\frac{\partial G}{\partial t}+\frac{1}{2} \sigma_{H}^{2} H^{2} G_{H H}+\frac{1}{2} \sigma_{x}^{2} G_{x x}+\frac{1}{2} \sigma_{y}^{2} G_{y y}+\sigma_{H x} H G_{H x}+\sigma_{H y} H G_{H y}+\sigma_{x y} G_{x y} \\
& +H G_{H} \bar{\mu}_{H}(t, x, y)+G_{x} \bar{\mu}_{x}(t, x, y)+G_{y} \bar{\mu}_{y}(t, x, y)-\bar{r}_{G}(t, x, y) G
\end{aligned}
$$


where $\tilde{k}=a^{\frac{1-\gamma}{\gamma}}\left(\frac{a R}{1-a}\right)^{k}$, and

$$
\begin{aligned}
-\bar{r}_{G}(t, x, y)= & \frac{\gamma-1}{\gamma} r(t, x, y)+\frac{\delta}{\gamma}+\frac{\gamma-1}{2 \gamma^{2}}\left[\left(\frac{\mu_{S}^{\prime}(t, x, y)+\chi_{S} x_{t}}{\sigma_{S} \hat{\rho}_{H}}\right)^{2}+\left(\frac{\mu_{H}^{\prime}(t, x, y)+\chi_{H x} x_{t}+\chi_{H y} y_{t}}{\sigma_{H} \hat{\rho}_{H}}\right)^{2}\right. \\
& \left.-2 \rho_{H S} \frac{\mu_{S}^{\prime}(t, x, y)+\chi_{S} x_{t}}{\sigma_{S} \hat{\rho}_{H}} \frac{\mu_{H}^{\prime}(t, x, y)+\chi_{H x} x_{t}+\chi_{H y} y_{t}}{\sigma_{H} \hat{\rho}_{H}}+\lambda_{L}(t, x, y)^{2}+\lambda_{x}(t, x, y)^{2}+\lambda_{y}(t, x, y)^{2}\right], \\
\bar{\mu}_{H}(t, x, y)= & r(t, x, y)-\frac{\gamma-1}{\gamma}[R-m]+\frac{1}{\gamma}\left(\mu_{H}(t, x, y)+\chi_{H x} x+\chi_{H y} y\right) \\
\bar{\mu}_{x}(t, x, y)= & -\left(\kappa_{x}+M_{x S} \chi_{S}+M_{x H} \chi_{H x}\right) x-M_{x H} \chi_{H y} y-M_{x S} \mu_{S}^{\prime}(t, x, y)-M_{x H} \mu_{H}^{\prime}(t, x, y) \\
& -\sigma_{x}\left(\hat{\rho}_{x L} \lambda_{L}(t, x, y)+\hat{\rho}_{x} \lambda_{x}(t, x, y)\right) \\
\bar{\mu}_{y}(t, x, y)= & -\left(M_{y S} \chi_{S}+M_{y H} \chi_{H x}\right) x-\left(\kappa_{y}+M_{y H} \chi_{H y}\right) y-M_{y S} \mu_{S}^{\prime}(t, x, y)-M_{y H} \mu_{H}^{\prime}(t, x, y) \\
& -\sigma_{y}\left(\hat{\rho}_{y L} \lambda_{L}(t, x, y)+\hat{\rho}_{y x} \lambda_{x}(t, x, y)+\hat{\rho}_{y} \lambda_{y}(t, x, y)\right) .
\end{aligned}
$$

The terminal condition is $G(T, H, x, y)=0$ because of the no-bequest assumption.

Coming back to the optimal investment strategy, we first note that

$$
\begin{aligned}
-\frac{J_{W}}{J_{W W}}=\frac{1}{\gamma}(W+L F), & -\frac{J_{W x}}{J_{W W}}=\frac{G_{x}}{G}(W+L F)-L F_{x}, \\
-\frac{J_{W L}}{J_{W W}}=-F, & -\frac{J_{W y}}{J_{W W}}=\frac{G_{y}}{G}(W+L F)-L F_{y}, \\
-\frac{J_{W H}}{J_{W W}}=\frac{G_{H}}{G}(W+L F) . &
\end{aligned}
$$

By substituting these expressions into (38)-(42), we obtain

$$
\begin{aligned}
\alpha_{S}= & \frac{1}{\gamma}(W+L F) \frac{1}{\sigma_{S} \hat{\rho}_{H}^{2}}\left(\mu_{S}^{\prime}(t, x, y)+\chi_{S} x-\frac{\rho_{H S} \sigma_{S}}{\sigma_{H}}\left[\mu_{H}^{\prime}(t, x, y)+\chi_{H x} x+\chi_{H y} y\right]\right)-F L M_{L S}(t) \sigma_{S} \\
& +\left[\frac{G_{x}}{G}(W+L F)-L F_{x}\right] M_{x S} \sigma_{S}+\left[\frac{G_{y}}{G}(W+L F)-L F_{y}\right] M_{y S} \sigma_{S}, \\
\alpha_{H}= & \frac{1}{\gamma}(W+L F) \frac{1}{\sigma_{H} \hat{\rho}_{H}^{2}}\left(\mu_{H}^{\prime}(t, x, y)+\chi_{H x} x+\chi_{H y} y-\frac{\rho_{H S} \sigma_{H}}{\sigma_{S}}\left[\mu_{S}^{\prime}(t, x, y)+\chi_{S} x\right]\right)+\frac{G_{H}}{G}(W+L F) H \sigma_{H} \\
& -F L M_{L H}(t) \sigma_{H}+\left[\frac{G_{x}}{G}(W+L F)-L F_{x}\right] M_{x H} \sigma_{H}+\left[\frac{G_{y}}{G}(W+L F)-L F_{y}\right] M_{y H} \sigma_{H}, \\
\alpha_{L}= & \frac{1}{\gamma}(W+L F) \lambda_{L}(t, x, y)-F L \hat{\rho}_{L} \sigma_{L}(t)+\left[\frac{G_{x}}{G}(W+L F)-L F_{x}\right] \hat{\rho}_{x L} \sigma_{x} \\
& +\left[\frac{G_{y}}{G}(W+L F)-L F_{y}\right] \hat{\rho}_{y L} \sigma_{y}, \\
\alpha_{x}= & \frac{1}{\gamma}(W+L F) \lambda_{x}(t, x, y)+\left[\frac{G_{x}}{G}(W+L F)-L F_{x}\right] \hat{\rho}_{x} \sigma_{x}+\left[\frac{G_{y}}{G}(W+L F)-L F_{y}\right] \hat{\rho}_{y x} \sigma_{y}, \\
\alpha_{y}= & \frac{1}{\gamma}(W+L F) \lambda_{y}(t, x, y)+\left[\frac{G_{y}}{G}(W+L F)-L F_{y}\right] \hat{\rho}_{y} \sigma_{y} .
\end{aligned}
$$


Below we show that $G(t, H, x, y)=\tilde{k} H^{k} B(t, x, y)$ so that

$$
\frac{G_{x}}{G}=\frac{B_{x}}{B}, \quad \frac{G_{y}}{G}=\frac{B_{y}}{B}, \quad \frac{H G_{H}}{G}=k .
$$

Then (25)-(29) in the theorem follows since $\Pi_{S}=\alpha_{S} /\left(\sigma_{S} W\right), \Pi_{H}=\alpha_{H} /\left(\sigma_{H} W\right), \Pi_{L}=\alpha_{L} / W, \Pi_{x}=\alpha_{x} / W$, and $\Pi_{y}=\alpha_{y} / W$.

If we substitute the conjecture $G(t, H, x, y)=\tilde{k} H^{k} B(t, x, y)$ into the PDE (44) and dividing by $\tilde{k} H^{k}$, we obtain

$$
0=1+\frac{\partial B}{\partial t}+\frac{1}{2} \sigma_{x}^{2} B_{x x}+\frac{1}{2} \sigma_{y}^{2} B_{y y}+\sigma_{x y} B_{x y}+\bar{\mu}_{x} B_{x}+\bar{\mu}_{y} B_{y}-\bar{r}_{G} B
$$

with terminal condition $B(T, x, y)=0$. Because of the complicated form of the coefficient functions (in particular $r(t, x, y))$, we solve the PDE (50) using standard finite difference techniques.

\section{B.4 Upper bound on obtainable utility}

The returns on the stock and house and the risk-free rate are at least as high in the artificial markets as in the true market. Therefore, a feasible strategy in the true market leads to at least the same expected utility in any of the artificial markets as in the true market. Since many other strategies are feasible in the artificial market, the indirect utility there is always greater or equal the indirect utility in the true market. Karatzas, Lehoczky, Shreve, and Xu (1991), Cvitanić and Karatzas (1992), and Cvitanić, Schachermayer, and Wang (2001) show that, under certain technical conditions, the solution in the true market is equal to the solution in the worst of all the artificial markets but, in complex models as our, it seems impossible to identify the worst market.

Theorem 1 provides a closed-form solution in any "computable" artificial market. The worst among these artificial markets defines an upper bound on the maximum expected utility in the true market. Each computable artificial market is parameterized by the constants appearing in (18)-(22). For easy reference, let $\Theta$ denote a set of such constants. We find the worst of the corresponding artificial markets by a standard unconstrained numerical optimization over $\Theta$. Let $\bar{\Theta}$ denote the parameter set for which the minimum is obtained. Hence,

$$
\bar{J}(t, W, H, L, x, y)=J(t, W, H, L, x, y ; \bar{\Theta})
$$

is the upper bound on the obtainable indirect utility in the true market (cf. Figure 3).

\section{B.5 Promising feasible strategies for the true problem}

We derive a promising strategy in the true market from the optimal strategies in the parameterized family of artificial markets in the following way. For each parameter set $\Theta$, we take the optimal strategy in the corresponding artificial market and feasibilize it, i.e., transform it into a strategy which is feasible in the 
true market. Obviously, we disregard the investment in the artificial asset and focus on $\Pi_{S}$, $\Pi_{H}$ and the consumption processes $c, \phi_{C}$.

In the artificial markets labor income is fully spanned and tangible wealth can be allowed to be temporarily negative if balanced by human capital. We require tangible wealth to stay non-negative because of the unhedgeable shocks that may bring income close to zero. We follow Bick, Kraft, and Munk (2013) and multiply the human capital by a factor $\left(1-e^{-\eta W_{t}}\right)$, where $\eta>0$ is a constant to be determined. This is consistent with the intuition that future income has a smaller present value when current wealth $W_{t}$ is small. Define

$$
\widetilde{F}_{t}=\left(1-e^{-\eta W_{t}}\right) F\left(t, x_{t}, y_{t}\right)
$$

Furthermore, we prune the optimal portfolios to make sure the constraints (10) are met. To sum up, the feasible strategy derived from the artificial market with parameters $\Theta$ is determined from

$$
\begin{aligned}
\Pi_{S t}= & \frac{1}{\gamma \hat{\rho}_{H}^{2} \sigma_{S}^{2}}\left(\mu_{S}^{\prime}(t, x, y)+\chi_{S} x_{t}-\frac{\rho_{H S} \sigma_{S}}{\sigma_{H}}\left[\mu_{H}^{\prime}(t, x, y)+\chi_{H x} x_{t}+\chi_{H y} y_{t}\right]\right) \frac{W_{t}+L_{t} \widetilde{F}_{t}}{W_{t}} \\
& +\left(M_{x S} \frac{B_{x}}{B}+M_{y S} \frac{B_{y}}{B}\right) \frac{W_{t}+L_{t} \widetilde{F}_{t}}{W_{t}}-\left(M_{L S}(t)+M_{x S} \frac{F_{x}}{F}+M_{x H} \frac{F_{y}}{F}\right) \frac{L_{t} \widetilde{F}_{t}}{W}, \\
\Pi_{H t}= & \frac{1}{\gamma \hat{\rho}_{H}^{2} \sigma_{H}^{2}}\left(\mu_{H}^{\prime}(t, x, y)+\chi_{H x} x_{t}+\chi_{H y} y_{t}-\frac{\rho_{H S} \sigma_{H}}{\sigma_{S}}\left[\mu_{S}^{\prime}(t, x, y)+\chi_{S} x_{t}\right]\right) \frac{W_{t}+L_{t} \widetilde{F}_{t}}{W_{t}} \\
& +k \frac{W_{t}+L_{t} \widetilde{F}_{t}}{W_{t}}+\left(M_{x H} \frac{B_{x}}{B}+M_{y H} \frac{B_{y}}{B}\right) \frac{W_{t}+L_{t} \widetilde{F}_{t}}{W_{t}}-\left(M_{L H}(t)+M_{x H} \frac{F_{x}}{F}+M_{y H} \frac{F_{y}}{F}\right) \frac{L_{t} \widetilde{F}_{t}}{W},
\end{aligned}
$$

and

$$
\begin{aligned}
c_{t} & =a \frac{W_{t}+L_{t} \widetilde{F}_{t}}{B\left(t, x_{t}, y_{t}\right)}, \\
\phi_{C t} & =(1-a) \frac{W_{t}+L_{t} \widetilde{F}_{t}}{R H_{t} B\left(t, x_{t}, y_{t}\right)},
\end{aligned}
$$

where we suppress the dependence of $F, F_{x}, F_{y}, B, B_{x}$, and $B_{y}$ on $t, x, y$ and the parameter set $\Theta$. If necessary to satisfy the portfolio constraints (10), we prune the portfolio weights $\Pi_{S}, \Pi_{H}$ as shown in Figure 4 . After these potential transformations, the residual wealth (positive or negative) constitutes the position in the bank account. If financial wealth should equal zero at any point in time, the investment in the risky assets is restricted to zero and consumption is set to fraction of current income, $c_{t}=\omega Y_{t}$ and $\phi_{C t}=\omega Y_{t} /\left(R H_{t}\right)$, where $\omega \in(0,1 / 2)$. This ensures that the liquidity constraint is respected.

For any $(\Theta, \eta)$, we can approximate the expected utility $J(t, W, H, L, x, y ; \Theta, \eta)$ generated with the above strategy by Monte Carlo simulation of the wealth $W_{t}$ and state variables $H_{t}, L_{t}, x_{t}, y_{t}$. Searching over $(\Theta, \eta)$, we find the best of the feasible strategies. This is our candidate for a near-optimal consumption-investment strategy in the true market. In Figure 3 this corresponds to the point marked $\Theta^{*}$ to the left of the unknown optimality point. Again, this search can be implemented by a standard unconstrained numerical optimization 
algorithm.

We can evaluate the performance of any admissible strategy $\left(c, \phi_{C}, \Pi_{S}, \Pi_{H}\right)$-including our candidate defined above - in the following way. We compare the expected utility generated by the strategy, $J^{c, \phi_{C}, \Pi_{S}, \Pi_{H}}(t, W, H, L, x, y)$, to the upper bound $\bar{J}(t, W, H, L, x, y)$ on the maximum utility. If the distance is small, the strategy is indeed near-optimal. More precisely, we can compute an upper bound

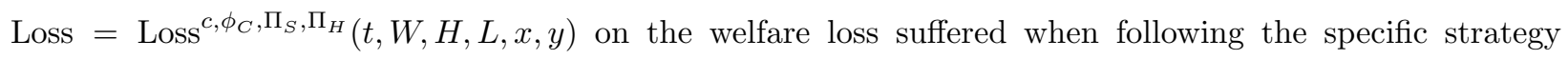
$\left(c, \phi_{C}, \Pi_{S}, \Pi_{H}\right)$ by solving the equation

$$
J^{c, \phi_{C}, \Pi_{S}, \Pi_{H}}(t, W, H, L, x, y)=\bar{J}(t, W[1-\operatorname{Loss}], H, L[1-\operatorname{Loss}], x, y) .
$$

We can interpret Loss as an upper bound on the fraction of total wealth (current wealth plus current and future income) that the individual is willing to sacrifice to get access to the unknown optimal strategy, instead of following the strategy $\left(c, \phi_{C}, \Pi_{S}, \Pi_{H}\right)$. Theorem 1 implies

$$
\begin{aligned}
\bar{J}(t, W[1-\mathrm{Loss}], H, L[1-\mathrm{Loss}], x, y) & =J(t, W[1-\mathrm{Loss}], H, L[1-\operatorname{Loss}], x, y ; \bar{\Theta}) \\
& =(1-\mathrm{Loss})^{1-\gamma} J(t, W, H, L, x, y ; \bar{\Theta}),
\end{aligned}
$$

so that the upper bound on the welfare loss becomes

$$
\operatorname{Loss}^{c, \phi_{C}, \Pi_{S}, \Pi_{H}}(t, W, H, L, x, y)=1-\left(\frac{J^{c, \phi_{C}, \Pi_{S}, \Pi_{H}}(t, W, H, L, x, y)}{J(t, W, H, L, x, y ; \bar{\Theta})}\right)^{\frac{1}{1-\gamma}} .
$$

\section{B.6 Solution in special cases}

To come: information on solution if $x$ or $y$ or both are ignored. 


\section{References}

Ang, A. and G. Bekaert (2007). Stock Return Predictability: Is It There? Review of Financial Studies 20(3), 651-707.

Barberis, N. (2000). Investing for the Long Run when Returns are Predictable. Journal of Finance 55(1), 225-264.

Bick, B., H. Kraft, and C. Munk (2013). Solving Constrained Consumption-Investment Problems by Simulation of Artificial Market Strategies. Management Science 59(2), 485-503.

Boudoukh, J., R. Michaely, M. Richardson, and M. R. Roberts (2007). On the Importance of Measuring Payout Yield: Implications for Empirical Asset Pricing. Journal of Finance 62(2), $877-916$.

Bourassa, S. C., D. R. Haurin, J. L. Haurin, M. Hoesli, and J. Sun (2009). House Price Changes and Idiosyncratic Risk: The Impact of Property Characteristics. Real Estate Economics 37(2), $259-278$.

Brown, S., W. Goetzmann, and S. A. Ross (1995). Survival. Journal of Finance 50(3), 853-873.

Campbell, J. Y. (2006). Household Finance. Journal of Finance 61(4), 1553-1604.

Campbell, J. Y. and R. J. Shiller (1988). The Dividend-Price Ratio and Expectations of Future Dividends and Discount Factors. Review of Financial Studies 1(3), 195-227.

Campbell, J. Y. and L. M. Viceira (1999). Consumption and Portfolio Decisions when Expected Returns are Time Varying. Quarterly Journal of Economics 114(2), 433-495.

Case, K. E. and R. J. Shiller (1989). The Efficiency of the Market for Single-Family Homes. American Economic Review 79(1), 125-137.

Case, K. E. and R. J. Shiller (1990). Forecasting Prices and Excess Returns in the Housing Market. Real Estate Economics 18(3), 253-273.

Cocco, J. F. (2005). Portfolio Choice in the Presence of Housing. Review of Financial Studies $18(2), 535-567$.

Cocco, J. F., F. J. Gomes, and P. J. Maenhout (2005). Consumption and Portfolio Choice over the Life Cycle. Review of Financial Studies 18(2), 491-533.

Corradin, S., J. L. Fillat, and C. Vergara-Alert (2014). Optimal Portfolio Choice with Predictability in House Prices and Transaction Costs. Review of Financial Studies 27(4), 823-880. 
Cotter, J. and R. Roll (2015). A Comparative Anatomy of Residential REITs and Private Real Estate Markets: Returns, Risks and Distributional Characteristics. Real Estate Economics 43(1), 209-240.

Cvitanić, J. and I. Karatzas (1992). Convex Duality in Constrained Portfolio Optimization. Annals of Applied Probability 2(4), 767-818.

Cvitanić, J., W. Schachermayer, and H. Wang (2001). Utility Maximization in Incomplete Markets with Random Endowment. Finance and Stochastics 5(2), 259-272.

Davis, M. A., A. Lehnert, and R. F. Martin (2008). The Rent-Price Ratio for the Aggregate Stock of Owner-Occupied Housing. Review of Income and Wealth 54(2), 279-284.

De Nardi, M., E. French, and J. B. Jones (2010). Why do the Elderly Save? The Role of Medical Expenses. Journal of Political Economy 118(1), 39-75.

Fama, E. F. and K. R. French (1988). Permanent and Temporary Components of Stock Prices. Journal of Political Economy 96(2), 246-273.

Fama, E. F. and K. R. French (2002). The Equity Premium. Journal of Finance 57(2), 637-659.

Fischer, M. and M. Stamos (2013). Optimal Life Cycle Portfolio Choice with Housing Market Cycles. Review of Financial Studies 26(9), 2311-2352.

Flavin, M. and T. Yamashita (2002). Owner-Occupied Housing and the Composition of the Household Portfolio. American Economic Review 91(1), 345-362.

Ghysels, E., A. Plazzi, R. Valkanov, and W. Torous (2013). Forecasting Real Estate Prices. In G. Elliott and A. Timmermann (Eds.), Handbook of Economic Forecasting, Volume 2, Part A, Chapter 9, pp. 509-580. Elsevier.

Guvenen, F., F. Karahan, S. Ozkan, and J. Song (2015, February). What Do Data on Millions of U.S. Workers Reveal About Life-Cycle Earnings Risk. Available at SSRN: http://ssrn. com/abstract $=2563279$.

van Hemert, O. (2010). Household Interest Rate Risk Management. Real Estate Economics 38(3), $467-505$.

Himmelberg, C., C. Mayer, and T. Sinai (2005). Assessing High House Prices: Bubbles, Fundamentals, and Misperseptions. Journal of Economic Perspectives 19(4), 67-92.

Hurd, M. D. (1989). Mortality Risk and Bequest. Econometrica 57(4), 779-813. 
Karatzas, I., J. Lehoczky, S. Shreve, and G. Xu (1991). Martingale and Duality Methods for Utility Maximization in an Incomplete Market. SIAM Journal on Control and Optimization 29(3), $702-730$.

Kim, T. S. and E. Omberg (1996). Dynamic Nonmyopic Portfolio Behavior. Review of Financial Studies 9(1), 141-161.

Koijen, R. S. J. and S. van Nieuwerburgh (2011). Predictability of Returns and Cash Flows. Annual Review of Financial Economics 3, 467-491.

Kraft, H. and C. Munk (2011). Optimal Housing, Consumption, and Investment Decisions over the Life-Cycle. Management Science 57(6), 1025-1041.

Lustig, H. and S. van Nieuwerburgh (2005). Housing Collateral, Consumption Insurance, and Risk Premia: An Empirical Perspective. Journal of Finance 60(3), 1167-1219.

Lynch, A. W. and S. Tan (2011). Labor Income Dynamics at Business-cycle Frequencies: Implications for Portfolio Choice. Journal of Financial Economics 101(2), 333-359.

Malpezzi, S. (1999). A Simple Error Correction Model of House Prices. Journal of Housing Economics 8(1), 27-62.

Moskowitz, T., Y. H. Ooi, and L. H. Pedersen (2012). Time Series Momentum. Journal of Financial Economics 104(2), 228-250.

Munk, C. and C. Sørensen (2010). Dynamic Asset Allocation with Stochastic Income and Interest Rates. Journal of Financial Economics 96(3), 433-462.

Plazzi, A., W. Torous, and R. Valkanov (2010). Expected Returns and Expected Growth in Rents of Commercial Real Estate. Review of Financial Studies 23(9), 3469-3519.

Poterba, J. M. (1991). House Price Dynamics: The Role of Tax Policy and Demography. Brookings Papers on Economic Activity 22(2), 143-203.

Santos, T. and P. Veronesi (2006). Labor Income and Predictable Stock Returns. Review of Financial Studies 19(1), 1-44.

Tsai, I.-C., M.-C. Chen, and T.-F. Sing (2007, November). Do REITs behave More like Real Estate Now? Available at http://ssrn.com/abstract=1079590.

U.S. Department of Labor, Bureau of Labor Statistics (2003). Consumer Expenditures in 2001. Report 966. 
Wachter, J. A. (2002). Portfolio and Consumption Decisions under Mean-Reverting Returns: An Exact Solution for Complete Markets. Journal of Financial and Quantitative Analysis 37(1), $63-91$.

Yao, R. and H. H. Zhang (2005). Optimal Consumption and Portfolio Choices with Risky Housing and Borrowing Constraints. Review of Financial Studies 18(1), 197-239. 


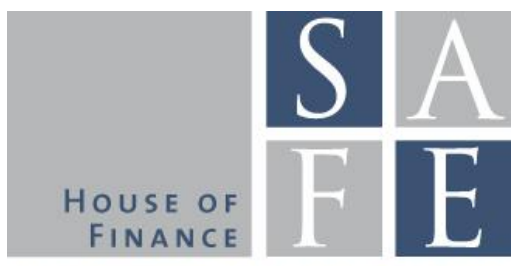

WORKING PAPER SERIES

\section{Recent Issues}

No. 138 Mohammed Aldegwy, Matthias Thiemann

No. 137

Elia Berdin, Cosimo Pancaro, Christoffer Kok

No. 136

Matthias Thiemann, Mohammed Aldegwy, Edin Ibrocevic

No. 135

No. 134

No. 133

No. 132

No. 131

No. 130

No. 129

No. 128

No. 127
Douglas Cumming, Jochen Werth, Yelin Zhang

Markus Kröll, Devesh Rustagi

Markus Behn, Rainer Haselmann, Thomas Kick, Vikrant Vig

Rainer Haselmann, David

Schoenherr, Vikrant Vig

Nicole Branger, Patrick Grüning, Christian Schlag

Giuliano Curatola

Giuliano Curatola, Michael Christoph Meinerding

Giuliano Curatola

Helmut Elsinger, Philipp SchmidtDengler, Christine Zulehner Donadelli, Patrick Grüning,
How Economics Got it Wrong: Formalism, Equilibrium Modelling and PseudoOptimization in Banking Regulatory Studies

A Stochastic Forward-Looking Model to Assess the Profitability and Solvency of European Insurers

Understanding the Shift from Micro to MacroPrudential Thinking: A Discursive Network Analysis

Governance in Entrepreneurial Ecosystems: Venture Capitalists vs. Technology Parks

Got Milk? Motivation for Honesty and Cheating in Informal Markets: Evidence from India

The Political Economy of Bank Bailouts

Rent-Seeking in Elite Networks

Commodities, Financialization, and Heterogeneous Agents

Optimal Consumption and Portfolio Choice with Loss Aversion

Investment-Specific Shocks, Business Cycles, and Asset Prices

Preference Evolution and the Dynamics of Capital Markets

Competition in Treasury Auctions 